\title{
PARTIALLY REFLECTED DIFFUSION
}

\author{
A. SINGER*, Z. SCHUSS ${ }^{\dagger}$, A. OSIPOV ${ }^{\ddagger}$, AND D. HOLCMAN $§$
}

\begin{abstract}
The radiation (reaction, Robin) boundary condition for the diffusion equation is widely used in chemical and biological applications to express reactive boundaries. The underlying trajectories of the diffusing particles are believed to be partially absorbed and partially reflected at the reactive boundary, however, the relation between the reaction constant in the Robin boundary condition and the reflection probability is not well defined. In this paper we define the partially reflected process as a limit of the Markovian jump process generated by the Euler scheme for the underlying Itô dynamics with partial boundary reflection. Trajectories that cross the boundary are terminated with probability $P \sqrt{\Delta t}$ and otherwise are reflected in a normal or oblique direction. We use boundary layer analysis of the corresponding master equation to resolve the non-uniform convergence of the probability density function of the numerical scheme to the solution of the Fokker-Planck equation in a half space, with the Robin constant $\kappa$. The boundary layer equation is of the Wiener-Hopf type. We show that the Robin boundary condition is recovered if and only if trajectories are reflected in the co-normal direction $\boldsymbol{\sigma n}$, where $\boldsymbol{\sigma}$ is the (possibly anisotropic) constant diffusion matrix and $\boldsymbol{n}$ is the unit normal to the boundary. Otherwise, the density satisfies an oblique derivative boundary condition. The constant $\kappa$ is related to $P$ by $\kappa=r P \sqrt{\sigma_{n}}$, where $r=1 / \sqrt{\pi}$ and $\sigma_{n}=\boldsymbol{n}^{T} \boldsymbol{\sigma n}$. The reflection law and the relation are new for diffusion in higher-dimensions.
\end{abstract}

Key words. stochastic differential equations, reactive boundary condition, Markovian jump process, Wiener-Hopf boundary layer equation

AMS subject classifications. 60H35, 60J50, $81 \mathrm{~S} 40$

1. Introduction. The Fokker-Planck equation (FPE) with radiation (also called reactive or Robin) boundary conditions is widely used to describe diffusion in a biological cell with chemical reactions on its surface [1, 2], 3], 4], [5], [6], [7, [8, 9]. The Robin boundary conditions are used in [2], 4], [5], 6] as a homogenization of mixed Dirichlet-Neumann boundary conditions given on scattered small absorbing windows in an otherwise reflecting boundary. The latter may represent, e.g., ligand binding or pumping out ions at sites on the boundary of a biological cell and no flux through the remaining boundary. The reactive rate constant in the Robin boundary conditions is chosen in the homogenization process so that the decay rate of the survival probability is the same as that in the mixed Dirichlet-Neumann boundary value problem.

The definition of the Itô stochastic dynamics

$$
\dot{x}=a(x, t)+\sqrt{2 \sigma(x, t)} \dot{w},
$$

on the positive axis with total or partial reflection at the origin was given first by Feller [10] for the one-dimensional case with $a(x, t)$ and $\sigma(x, t)$ independent of $t$, as a limit of Itô processes, which are terminated when they reach the boundary or moved instantaneously to a point $x=\rho_{j}>0$ with probability $p_{j}$. When $p_{j} \rightarrow 1$ and $\rho_{j} \rightarrow 0$ with

$$
\lim _{j \rightarrow \infty} \frac{1-p_{j}}{\rho_{j}}=c,
$$

where $c$ is a constant, the partially reflected process converges to a limit. The transition probability density function (pdf) of the limit process, $p(y, t \mid x, s) d y=\operatorname{Pr}\{x(t) \in(y, y+d y) \mid x(s)=x\}$,

${ }^{*}$ Department of Mathematics, Program in Applied Mathematics, Yale University, 10 Hillhouse Ave. PO Box 208283, New Haven, CT 06520-8283 (amit.singer@yale.edu)

${ }^{\dagger}$ Department of Applied Mathematics, Tel-Aviv University, Ramat-Aviv 69978 Tel-Aviv, Israel (schuss@post.tau.ac.il). The research of this author was partially supported by a grant from TAU.

${ }^{\ddagger}$ Institute of Mathematics, The Hebrew University, Jerusalem 91904, Israel (andreiosipov2@gmail.com)

$\S$ Department of Mathematics, Weizmann Institute of Science, Rehovot 76100 Israel (david.holcman@weizmann.ac.il). This author is incumbent to the Madeleine Haas Russell Career Development Chair. 
is the solution of the FPE

$$
\frac{\partial p(y, t \mid x, s)}{\partial t}=-\frac{\partial[a(y, t) p(y, t \mid x, s)]}{\partial y}+\frac{\partial^{2}[\sigma(y, t) p(y, t \mid x, s)]}{\partial y^{2}},
$$

or equivalently,

$$
\frac{\partial p(y, t \mid x, s)}{\partial t}=-\frac{\partial J(y, t \mid x, s)}{\partial y} \text { for all } y, x>0
$$

where

$$
J(y, t \mid x, s)=a(y, t) p(y, t \mid x, s)-\frac{\partial[\sigma(y, t) p(y, t \mid x, s)]}{\partial y},
$$

is the flux. The initial condition is

$$
p(y, t \mid x, s) \rightarrow \delta(y-x) \quad \text { as } \quad t \downarrow s
$$

and the radiation boundary condition is

$$
-J(0, t \mid x, s)=\kappa p(0, t \mid x, s),
$$

where $\kappa$ is a constant related to the constant $c$ and to the values of the coefficients at the boundary. The no flux and Dirichlet boundary conditions are recovered if $c=0$ or $c=\infty$, respectively. Feller's method does not translate into a Brownian dynamics simulation of the limit process, because his approximations are continuous-time Itô processes. Skorokhod [11 defines the reflection process inside the boundary. Several numerical schemes have been proposed for simulating this process (see, e.g., [11, [12, [13], [14]). The main issue there is to approximate the local time spent on the boundary.

The definition of a diffusion process with absorbing or reflecting boundaries as limits of Markovian jump processes, which is the basis for all simulations, gives in the limit diffusion processes with well defined boundary behavior. However, the definition of a diffusion process with partially reflecting boundaries as a limit of Markovian jump processes gives different diffusions for different jump processes. This is expressed in different relations between the termination probability of the jump process and the boundary conditions for the FokkerPlanck equations (see, e.g., [8]). The process $x(t)$ defined by equation (1.1) with partially absorbing boundaries can be defined as the limit of the solutions of the Markovian jump processes generated by the Euler scheme

$$
\begin{aligned}
x_{\Delta t}(t+\Delta t) & =x_{\Delta t}(t)+a\left(x_{\Delta t}(t), t\right) \Delta t+\sqrt{2 \sigma\left(x_{\Delta t}(t), t\right)} \Delta w(t, \Delta t) \quad \text { for } t \geq s \\
x_{\Delta t}(s) & =x
\end{aligned}
$$

in the interval $x>0$, for $0 \leq t-s \leq T$, with $\Delta t=T / N, t-s=i T / N(i=0,1, \ldots, N)$, where for each $t$ the random variables $\Delta w(t, \Delta t)$ are normally distributed and independent with zero mean and variance $\Delta t$. The partially absorbing boundary condition for (1.7) has to be chosen so that the pdf $p_{\Delta t}(x, t)$ of $x_{\Delta t}(t)$ converges to the solution of (1.3)-(1.6). At a partially reflecting boundary for (1.7), the trajectories are reflected with probability (w.p.) $R$ and otherwise terminated (absorbed), once they cross the origin. We show below that keeping $R$ constant (e.g., $R=1 / 2$ ) as $\Delta t \rightarrow 0$ leads to the convergence of the pdf $p_{\Delta t}(x, t)$ to the solution of the FPE with an absorbing rather than the Robin boundary condition. Thus the reflection probability $R$ must increase to 1 as $\Delta t \rightarrow 0$ in order to yield the Robin condition (1.6). Moreover, the reactive constant $\kappa$ is related to the limit

$$
\lim _{\Delta t \rightarrow 0} \frac{1-R}{\sqrt{\Delta t}}=P .
$$


The reflecting boundary condition is recovered for $P=0$, while the absorbing boundary condition is obtained for $P=\infty$. Motivated by these considerations, we design the following simple boundary behavior for the simulated trajectories that cross the boundary, identified by $x_{\Delta t}(t)+a\left(x_{\Delta t}(t), t\right) \Delta t+\sqrt{2 \sigma\left(x_{\Delta t}(t), t\right)} \Delta w<0$,

$$
x_{\Delta t}(t+\Delta t)= \begin{cases}-\left(x_{\Delta t}(t)+a\left(x_{\Delta t}(t), t\right) \Delta t+\sqrt{2 \sigma\left(x_{\Delta t}(t), t\right)} \Delta w\right) \quad \text { w.p. } 1-P \sqrt{\Delta t} \\ \text { terminate trajectory otherwise. }\end{cases}
$$

The exiting trajectory is normally reflected w.p.

$$
R=1-P \sqrt{\Delta t}
$$

and is otherwise terminated (absorbed). The scaling of the termination probability with $\sqrt{\Delta t}$ reflects the fact that the discrete unidirectional diffusion current at any point, including the boundary, is $O(1 / \sqrt{\Delta t})$ (see [15, [16]). This means that the number of discrete trajectories hitting or crossing the boundary in any finite time interval increases as $1 / \sqrt{\Delta t}$. Therefore, to keep the efflux of trajectories finite as $\Delta t \rightarrow 0$, the termination probability of a crossing trajectory, $1-R$, has to be $O(\sqrt{\Delta t})$. The pdf $p_{\Delta t}(x, t)$, however, does not converge to the solution $p(x, t)$ of (1.3)-(1.6) on the boundary, as discussed in Section 2. This is due to the formation of a boundary layer, as is typical for diffusion approximations of Markovian jump processes that jump over the boundary [17], [18], [19. The boundary layer equations are typically Wiener-Hopf integral equations. The Wiener-Hopf boundary layer equation for the particular case of a partially reflected Brownian motion on the positive axis (i.e., $a(x, t)=0$ and $\sigma(x, t)=\sigma$ in (1.7) $)$ was recently solved in [8] and the relationship $\kappa=P \sqrt{\sigma} / \sqrt{\pi}$ was found.

The convergence of the pdf of an Euler scheme has been studied in 20, 21] for the higher-dimensional problem with oblique reflection. Bounds on the integral norm of the approximation error are given for the solution of the backward Kolmogorov equation. These, however, do not resolve the boundary layer of the pdf of the numerical solution. The solution of the forward equation for the Euler scheme converges non-uniformly to the solution of the Fokker-Planck equation due to the appearance of a boundary layer in the first order spatial derivative. This distorts the boundary flux and gives incorrect boundary conditions. A boundary layer expansion is needed to capture the boundary phenomena.

The derivation of the radiation condition has a long history. Collins and Kimball 22. (see also [23) derived the radiation boundary condition (1.6) for the limit $p(x, t)=$ $\lim _{\Delta t \rightarrow 0} p_{\Delta t}(x, t)$ from an underlying discrete random walk model on a semi-infinite onedimensional lattice with partial absorbtion at the endpoint. Their model assumes constant diffusion coefficient and vanishing drift, for which they find the reactive constant in terms of the absorbtion probability and the diffusion coefficient. Previous simulation schemes that recover the Robin boundary condition [1], 24], 25, 26], 27] make use of the explicit solution to the half space FPE with linear drift term and constant diffusion coefficient with a Robin condition. In [28, and references therein] the specular reflection method near a reflecting boundary has been shown to be superior to other methods, such as rejection, multiple rejection and interruption.

An apparent paradox arises when using (1.7) and other schemes: while the pdf $p_{\Delta t}(y, t \mid x, s)$ of the solution of (1.7), (1.8), (1.10), (1.11) converges to the solution of the FPE (1.3) and the initial condition (1.5), each approximant $p_{\Delta t}(y, t \mid x, s)$ does not satisfy the boundary condition (1.6), not even approximately, that is, the error does not decay as $\Delta t \rightarrow 0$. For a general diffusion coefficient and drift term, the boundary condition is not satisfied even for the case of a reflecting boundary condition. This problem plagues other schemes as well. The apparent paradox is due to the non-uniform convergence of $p_{\Delta t}(y, t \mid x, s)$ to the solution 
$p(y, t \mid x, s)$ of the Fokker-Planck equation, caused by a boundary layer in $p_{\Delta t}(y, t \mid x, s)$, as is typical of boundary behavior of diffusion approximations to Markovian jump processes. The limit $p(y, t \mid x, s)$, however, satisfies the boundary condition (1.6) for some $\kappa$. Our analysis can be extended to other schemes in a straightforward way. It is well known that the Euler scheme produces an $O(\sqrt{\Delta t})$ error in estimating the mean first passage time to reach an absorbing boundary. There are several recipes to reduce the discretization error to $O(\Delta t)$ [29], [30], 31, 32, 33. Another manifestation of the boundary layer is that the approximation error of the pdf near absorbing or reflecting boundaries is $O(\sqrt{\Delta t})$, and methods, including [1], 34 reduce this error to $O(\Delta t)$. Thus, we expect the formation of a boundary layer of size $O(\sqrt{\Delta t})$ for the Euler scheme (1.7) with the boundary behavior (1.10).

This paper is concerned with the convergence of the partially reflecting Markovian jump process generated by (1.7), (1.10) in one and higher dimensions. We show that this scheme, with the additional requirement that the pdf converges to the solution of the FPE with a given Robin boundary condition, defines a unique diffusion process with partial reflection at the boundary. This definition is then generalized to higher dimensions. In contrast to the Collins and Kimball 22] discrete scheme, this definition is not restricted to lattice points and the drift and diffusion coefficients may vary. The advantage of the current suggested design (1.10) is its simplicity, which is both easily and efficiently implemented and amenable to analysis. There is no need to make any assumptions on the structure of the diffusion coefficient or the drift. From the theoretical point of view, it serves as a physical interpretation for the behavior of diffusive trajectories near a reactive boundary.

Our main result in the one-dimensional case is the relation between the reactive "constant" $\kappa(t)$ and the absorbtion parameter $P$ for the dynamics (1.1) on the positive axis with drift and with a variable diffusion coefficient,

$$
\kappa(t)=r P \sqrt{\sigma(0, t)}, \quad r=\frac{1}{\sqrt{\pi}}
$$

The relation (1.12) is new for diffusion with variable coefficients. The value $r=1 / \sqrt{\pi}$ is different than values obtained for other schemes, e.g., than the value $r=1 / \sqrt{2}$, predicted by the discrete random walk theory of radiation boundaries [22. Values of $r$ for other schemes are given in 8 . We show the effect of using (1.12) in numerical simulations.

The scheme (1.10) is generalized to diffusion with drift and anisotropic constant diffusion matrix $\boldsymbol{\sigma}(t)$ in the half space, $x_{1}>0$, with partial oblique reflection. We show that the Robin boundary condition is recovered if and only if trajectories are reflected in the direction of the unit vector

$$
v=\frac{\sigma n}{\|\sigma n\|}
$$

where $\boldsymbol{n}$ is the unit normal to the boundary. The radiation parameter $\kappa(\boldsymbol{x}, t)$ in the $d$ dimensional Robin boundary condition and the absorbtion parameter $P(\boldsymbol{x})$ are related by

$$
\kappa(\boldsymbol{x}, t)=r P(\boldsymbol{x}) \sqrt{\sigma_{n}(t)}, \quad x_{1}=0,
$$

with $r$ given in (1.12) and $\sigma_{n}(t)=\boldsymbol{n}^{T} \boldsymbol{\sigma}(t) \boldsymbol{n}$. The relation (1.14) is new for higherdimensional diffusion in a half space with drift and anisotropic diffusion matrix.

In the most common case of constant isotropic diffusion our result extends to domains with curved boundaries. This is due to the fact that a smooth local mapping of the domain to a half space with an orthogonal system of coordinates preserves the constant isotropic diffusion matrix, though the drift changes according to Itô's formula. In this case the vector $\boldsymbol{v}$ coincides with the normal $\boldsymbol{n}$. 
2. Boundary layer analysis in one dimension. The aim of the boundary layer analysis below is to examine the convergence of the pdf $p_{\Delta t}(y, t \mid x, s)$ of the solution $x_{\Delta t}(t)$ of (1.7), (1.8) to the solution $p(y, t \mid x, s)$ of (1.3)-(1.6), and to find the relation between the parameter $P$ of (1.10) and the reactive constant $\kappa$ in (1.6). Using abbreviated notation, the pdf $p_{\Delta t}(y, t \mid x, s)=p_{\Delta t}(y, t)$ satisfies the forward Kolmogorov equation [15, 16, 17, [18, 19], 35]

$$
\begin{aligned}
p_{\Delta t}(y, t+\Delta t)= & \int_{0}^{\infty} \frac{p_{\Delta t}(x, t)}{\sqrt{4 \pi \sigma(x, t) \Delta t}}\left\{\exp \left[-\frac{(y-x-a(x, t) \Delta t)^{2}}{4 \sigma(x, t) \Delta t}\right]+\right. \\
& \left.(1-P \sqrt{\Delta t}) \exp \left[-\frac{(y+x+a(x, t) \Delta t)^{2}}{4 \sigma(x, t) \Delta t}\right]\right\} d x .
\end{aligned}
$$

For $P=0$ the pdf $p_{\Delta t}(y, t)$ satisfies the boundary condition

$$
\frac{\partial p_{\Delta t}(0, t)}{\partial y}=0
$$

which is obtained by differentiation of (2.1) with respect to $y$ at $y=0$. If $P \neq 0$, we obtain

$$
\frac{\partial p_{\Delta t}(0, t+\Delta t)}{\partial y}=\frac{p_{\Delta t}(0, t) P}{\sqrt{4 \pi \sigma(0, t)}}+O(\sqrt{\Delta t})
$$

which holds also in the limit $\Delta t \rightarrow 0$. However, the order of the limits $\Delta t \rightarrow 0$ and $y \downarrow 0$ matters, indeed,

$$
\lim _{\Delta t \rightarrow 0} \lim _{y \downarrow 0} \frac{\partial p_{\Delta t}(y, t)}{\partial y} \neq \lim _{y \downarrow 0} \lim _{\Delta t \rightarrow 0} \frac{\partial p_{\Delta t}(y, t)}{\partial y} .
$$

The limit of (2.3) is not the boundary condition that the limit function $p(y, t)=\lim _{\Delta t \rightarrow 0} p_{\Delta t}(y, t)$ (for $y>0$ ) satisfies. To find the boundary condition of $p(y, t)$, in either case $P=0$ or $P \neq 0$, we show below that $p(y, t)$ satisfies the FPE (1.3) and the initial condition (1.5) for all $y>0$. Since for $P=0$ the simulation preserves probability (the population of trajectories),

$$
0=\frac{d}{d t} \int_{0}^{\infty} p(x, t) d x=-\frac{\partial[\sigma(0, t) p(0, t)]}{\partial y}+a(0, t) p(0, t)=J(0, t) .
$$

Equation (2.5) is the no-flux boundary condition. The discrepancy between (2.5) and (2.2) is due to the nonuniform convergence of $p_{\Delta t}(y, t)$ to its limit $p(y, t)$ in the interval. There is a boundary layer of width $O(\sqrt{\Delta t})$, in which the boundary condition (2.2) for $p_{\Delta t}(y, t)$ changes into the boundary condition (2.5) that $p(y, t)$ satisfies. To analyze the discrepancy between (2.2) and (2.5), we introduce the local variable $y=\eta \sqrt{\Delta t}$ and the boundary layer solution

$$
p_{B L}(\eta, t)=p_{\Delta t}(\eta \sqrt{\Delta t}, t) .
$$

Changing variables $x=\xi \sqrt{\Delta t}$ in the integral (2.1) gives

$$
\begin{aligned}
& p_{B L}(\eta, t+\Delta t)= \int_{0}^{\infty} \frac{p_{B L}(\xi, t)}{\sqrt{4 \pi \sigma(\xi \sqrt{\Delta t}, t)}}\left\{\exp \left[-\frac{(\eta-\xi-a(\xi \sqrt{\Delta t}, t) \sqrt{\Delta t})^{2}}{4 \sigma(\xi \sqrt{\Delta t}, t)}\right]+\right. \\
&\left.(1-P \sqrt{\Delta t}) \exp \left[-\frac{(\eta+\xi+a(\xi \sqrt{\Delta t}, t) \sqrt{\Delta t})^{2}}{4 \sigma(\xi \sqrt{\Delta t}, t)}\right]\right\} d \xi \\
& 5
\end{aligned}
$$


The boundary layer solution has an asymptotic expansion in powers of $\sqrt{\Delta t}$

$$
p_{B L}(\eta, t) \sim p_{B L}^{(0)}(\eta, t)+\sqrt{\Delta t} p_{B L}^{(1)}(\eta, t)+\Delta t p_{B L}^{(2)}(\eta, t)+\ldots
$$

Expanding all functions in (2.7) in powers of $\sqrt{\Delta t}$ and equating similar orders, we obtain integral equations that the asymptotic terms of (2.8) must satisfy. The leading order $O(1)$ term gives the Wiener-Hopf-type equation on the half line

$$
p_{B L}^{(0)}(\eta, t)=\int_{0}^{\infty} \frac{p_{B L}^{(0)}(\xi, t)}{\sqrt{4 \pi \sigma(0, t)}}\left\{\exp \left[-\frac{(\eta-\xi)^{2}}{4 \sigma(0, t)}\right]+\exp \left[-\frac{(\eta+\xi)^{2}}{4 \sigma(0, t)}\right]\right\} d \xi,
$$

for $\eta>0$. The kernel

$$
K(\eta, \xi)=\exp \left[-\frac{(\eta-\xi)^{2}}{4 \sigma(0, t)}\right]+\exp \left[-\frac{(\eta+\xi)^{2}}{4 \sigma(0, t)}\right]
$$

is an even function of $\eta$ and $\xi$, i.e. $K(\eta, \xi)=K(-\eta, \xi)=K(\eta,-\xi)=K(-\eta,-\xi)$. Therefore, we extend $p_{B L}^{(0)}(\xi, t)$ to the entire line as an even function $\left(p_{B L}^{(0)}(\xi, t)=p_{B L}^{(0)}(-\xi, t)\right)$, and rewrite (2.9) as

$$
p_{B L}^{(0)}(\eta, t)=\int_{-\infty}^{\infty} \frac{p_{B L}^{(0)}(\xi, t)}{\sqrt{4 \pi \sigma(0, t)}} \exp \left[-\frac{(\eta-\xi)^{2}}{4 \sigma(0, t)}\right] d \xi,
$$

for $-\infty<\eta<\infty$. The only solution of the integral equation (2.11) is the constant function, that is, $p_{B L}^{(0)}(\eta, t)=f(t)$, independent of $\eta$. This follows immediately from the Fourier transform of (2.11), whose right hand side is a convolution.

Away from the boundary layer the solution admits an outer solution expansion

$$
p_{O U T}(y, t) \sim p_{O U T}^{(0)}(y, t)+\sqrt{\Delta t} p_{O U T}^{(1)}(y, t)+\ldots,
$$

where $p_{\text {OUT }}^{(0)}$ satisfies the Fokker-Planck equation (1.3) and the initial condition (1.5). Indeed, the integrals in (2.1) are of Laplace type with the small parameter $\Delta t$. For interior points $y \gg \sqrt{\Delta t}$ the second integral, which represents only boundary interactions, is negligible relative to the first one. We change variables in (2.1) by setting

$$
\eta=\frac{y-x-a(x, t) \Delta t}{\sqrt{2 \sigma(x, t) \Delta t}}
$$

and extend integration over the entire line in the first integral and expand all functions in powers of $\sqrt{\Delta t}$. The resulting integrals are moments of the normal distribution. We obtain

$$
\frac{p_{\Delta t}(y, t+\Delta t)-p_{\Delta t}(y, t)}{\Delta t}=-\frac{\partial\left[a(y, t) p_{\Delta t}(y, t)\right]}{\partial y}+\frac{\partial^{2}\left[\sigma(y, t) p_{\Delta t}(y, t)\right]}{\partial y^{2}}+O(\sqrt{\Delta t}) .
$$

The leading term in the expansion of $p_{\Delta t}(y, t)$ is $p_{O U T}^{(0)}(y, t)$, which therefore satisfies the Fokker-Planck equation (1.3). The initial condition (1.5) is recovered from the Gaussian integral as $\Delta t \rightarrow 0$. The boundary condition that $p_{O U T}^{(0)}(y, t)$ satisfies can be determined only after the boundary layer is resolved by matching. The leading order matching condition of the boundary layer and the outer solutions is

$$
\lim _{\eta \rightarrow \infty} p_{B L}^{(0)}(\eta, t)=p_{O U T}^{(0)}(0, t) .
$$

Therefore

$$
p_{B L}^{(0)}(\eta, t)=p_{O U T}^{(0)}(0, t) .
$$


The matching condition at order $\sqrt{\Delta t}$ gives

$$
\eta \frac{\partial p_{O U T}^{(0)}(0, t)}{\partial y}+p_{O U T}^{(1)}(0, t) \sim p_{B L}^{(1)}(\eta, t) \quad \text { for } \quad \eta \rightarrow \infty
$$

which means that $p_{B L}^{(1)}(\eta, t)$ is asymptotically a linear function of $\eta$, therefore the limit of its derivative is a constant. Thus the matching condition reduces to

$$
\lim _{\eta \rightarrow \infty} \frac{\partial p_{B L}^{(1)}(\eta, t)}{\partial \eta}=\frac{\partial p_{O U T}^{(0)}(0, t)}{\partial y} .
$$

The first order boundary layer term satisfies the integral equation

$$
\begin{aligned}
& p_{B L}^{(1)}(\eta, t)=\int_{0}^{\infty} \frac{p_{B L}^{(1)}(\xi, t)}{\sqrt{4 \pi \sigma(0, t)}}\left\{\exp \left[-\frac{(\eta-\xi)^{2}}{4 \sigma(0, t)}\right]+\exp \left[-\frac{(\eta+\xi)^{2}}{4 \sigma(0, t)}\right]\right\} d \xi- \\
& P \int_{0}^{\infty} \frac{p_{B L}^{(0)}(\xi, t)}{\sqrt{4 \pi \sigma(0, t)}} \exp \left[-\frac{(\eta+\xi)^{2}}{4 \sigma(0, t)}\right] d \xi- \\
& \frac{\sigma_{y}(0, t)}{2 \sigma(0, t)} \int_{0}^{\infty} \frac{p_{B L}^{(0)}(\xi, t)}{\sqrt{4 \pi \sigma(0, t)}} \xi\left\{\exp \left[-\frac{(\eta-\xi)^{2}}{4 \sigma(0, t)}\right]+\exp \left[-\frac{(\eta+\xi)^{2}}{4 \sigma(0, t)}\right]\right\} d \xi+ \\
& \frac{\sigma_{y}(0, t)}{4 \sigma(0, t)^{2}} \int_{0}^{\infty} \frac{p_{B L}^{(0)}(\xi, t)}{\sqrt{4 \pi \sigma(0, t)}} \xi\left\{(\eta-\xi)^{2} \exp \left[-\frac{(\eta-\xi)^{2}}{4 \sigma(0, t)}\right]+(\eta+\xi)^{2} \exp \left[-\frac{(\eta+\xi)^{2}}{4 \sigma(0, t)}\right]\right\} d \xi+ \\
& \frac{2 a(0, t)}{4 \sigma(0, t)} \int_{0}^{\infty} \frac{p_{B L}^{(0)}(\xi, t)}{\sqrt{4 \pi \sigma(0, t)}}\left\{(\eta-\xi) \exp \left[-\frac{(\eta-\xi)^{2}}{4 \sigma(0, t)}\right]-(\eta+\xi) \exp \left[-\frac{(\eta+\xi)^{2}}{4 \sigma(0, t)}\right]\right\} d \xi
\end{aligned}
$$

Evaluating explicitly the last four integrals in (2.15) and using (2.13), gives

$$
\begin{aligned}
p_{B L}^{(1)}(\eta, t)= & \int_{0}^{\infty} \frac{p_{B L}^{(1)}(\xi, t)}{\sqrt{4 \pi \sigma(0, t)}}\left\{\exp \left[-\frac{(\eta-\xi)^{2}}{4 \sigma(0, t)}\right]+\exp \left[-\frac{(\eta+\xi)^{2}}{4 \sigma(0, t)}\right]\right\} d \xi- \\
& \frac{P}{2} p_{O U T}^{(0)}(0, t) \operatorname{erfc}\left(\frac{\eta}{2 \sqrt{\sigma(0, t)}}\right)+\frac{\sigma_{y}(0, t)-a(0, t)}{\sqrt{\pi \sigma(0, t)}} p_{O U T}^{(0)}(0, t) \exp \left[-\frac{\eta^{2}}{4 \sigma(0, t)}\right] .
\end{aligned}
$$

Differentiating (2.16) with respect to $\eta$ and integrating by parts, we obtain

$$
\begin{aligned}
& \frac{\partial p_{B L}^{(1)}(\eta, t)}{\partial \eta}=\frac{1}{\sqrt{4 \pi \sigma(0, t)}} \int_{0}^{\infty} \frac{\partial p_{B L}^{(1)}(\xi, t)}{\partial \eta}\left\{\exp \left[-\frac{(\eta-\xi)^{2}}{4 \sigma(0, t)}\right]-\exp \left[-\frac{(\eta+\xi)^{2}}{4 \sigma(0, t)}\right]\right\} d \xi+ \\
& \frac{P}{2 \sqrt{\pi \sigma(0, t)}} p_{O U T}^{(0)}(0, t) \exp \left[\frac{-\eta^{2}}{4 \sigma(0, t)}\right]-\frac{\sigma_{y}(0, t)-a(0, t)}{2 \sqrt{\pi} \sigma(0, t)^{3 / 2}} p_{O U T}^{(0)}(0, t) \eta \exp \left[\frac{-\eta^{2}}{4 \sigma(0, t)}\right] \cdot(2.17)
\end{aligned}
$$

Setting

$$
g(\eta, t)=\frac{\partial p_{B L}^{(1)}(\eta, t)}{\partial \eta}-\frac{P}{2 \sqrt{\pi \sigma(0, t)}} p_{O U T}^{(0)}(0, t) \exp \left[-\frac{\eta^{2}}{4 \sigma(0, t)}\right],
$$

we rewrite (2.17) as

$$
\begin{aligned}
& g(\eta, t)= \\
& \phi(\eta, t)+\frac{1}{\sqrt{4 \pi \sigma(0, t)}} \int_{0}^{\infty} g(\xi, t)\left\{\exp \left[-\frac{(\eta-\xi)^{2}}{4 \sigma(0, t)}\right]-\exp \left[-\frac{(\eta+\xi)^{2}}{4 \sigma(0, t)}\right]\right\} d \xi,
\end{aligned}
$$


where

$$
\begin{aligned}
\phi(\eta, t)= & \frac{P}{\sqrt{8 \pi \sigma(0, t)}} p_{O U T}^{(0)}(0, t) \exp \left[\frac{-\eta^{2}}{8 \sigma(0, t)}\right] \operatorname{erf}\left(\frac{\eta}{\sqrt{8 \sigma(0, t)}}\right)- \\
& \frac{\sigma_{y}(0, t)-a(0, t)}{2 \sqrt{\pi} \sigma(0, t)^{3 / 2}} p_{O U T}^{(0)}(0, t) \eta \exp \left[\frac{-\eta^{2}}{4 \sigma(0, t)}\right] .
\end{aligned}
$$

Since $\phi(\eta, t)$ is an odd function of $\eta$, we can define $g(\eta, t)$ for negative values as an odd function by setting $g(\eta, t)=-g(-\eta, t)$ for $\eta<0$. Then (2.19) can be rewritten as

$$
g(\eta, t)=\phi(\eta, t)+\frac{1}{\sqrt{4 \pi \sigma(0, t)}} \int_{-\infty}^{\infty} g(\xi, t) \exp \left[-\frac{(\eta-\xi)^{2}}{4 \sigma(0, t)}\right] d \xi
$$

which in Fourier space is

$$
\hat{g}(k, t)=\frac{\hat{\phi}(k, t)}{1-\exp \left[-\sigma(0, t) k^{2}\right]} .
$$

Using the Wiener-Hopf method, we decompose

$$
\hat{g}(k, t)=\hat{g}_{+}(k, t)+\hat{g}_{-}(k, t),
$$

where $g_{+}(\eta)=g(\eta) \chi_{[0, \infty)}(\eta), g_{-}(\eta)=g(\eta) \chi_{(-\infty, 0]}(\eta)$. The Fourier transform $\hat{g}(k, t)$ exists in the sense of distributions, and $\hat{g}_{ \pm}(k, t)$ are analytic in the upper and lower halves of the complex plane, respectively. Taylor's expansion of $\hat{\phi}(k, t)$ in eq.(2.20) gives

$$
\hat{\phi}(k, t)=2 i p_{O U T}^{(0)}(0, t)\left\{\frac{P \sqrt{\sigma(0, t)}}{\sqrt{\pi}}-\left[\sigma_{y}(0, t)-a(0, t)\right]\right\} k+O\left(k^{3}\right) \quad \text { as } \quad k \rightarrow 0 .
$$

The the non-zero poles of (2.22) split evenly between $\hat{g}_{+}(k, t)$ and $\hat{g}_{-}(k, t)$, and using $\hat{g}_{+}(k, t)=-\hat{g}_{-}(-k, t)$, the pole at the origin gives

$$
\hat{g}_{+}(k, t)=i p_{\text {OUT }}^{(0)}(0, t)\left\{\frac{P}{\sqrt{\pi \sigma(0, t)}}-\frac{\sigma_{y}(0, t)-a(0, t)}{\sigma(0, t)}\right\} \frac{1}{k}+O(k) \quad \text { as } \quad k \rightarrow 0 .
$$

Inverting the Fourier transform $\hat{g}_{+}(k, t)$, by closing the contour of integration around the lower half plane, we obtain

$$
\lim _{\eta \rightarrow \infty} \frac{\partial p_{B L}^{(1)}(\eta, t)}{\partial \eta}=p_{O U T}^{(0)}(0, t)\left\{\frac{P}{\sqrt{\pi \sigma(0, t)}}-\frac{\sigma_{y}(0, t)-a(0, t)}{\sigma(0, t)}\right\} .
$$

The matching condition (2.14) implies

$$
\frac{\partial p_{O U T}^{(0)}(0, t)}{\partial y}=p_{O U T}^{(0)}(0, t)\left\{\frac{P}{\sqrt{\pi \sigma(0, t)}}-\frac{\sigma_{y}(0, t)-a(0, t)}{\sigma(0, t)}\right\} .
$$

Multiplying by $\sigma(0, t)$ and rearranging, we obtain the radiation boundary condition

$$
-J(0, t)=\frac{\partial}{\partial y}\left[\sigma(0, t) p_{O U T}^{(0)}(0, t)\right]-a(0, t) p_{O U T}^{(0)}(0, t)=\frac{P \sqrt{\sigma(0, t)}}{\sqrt{\pi}} p_{O U T}^{(0)}(0, t) .
$$

Since $p(y, t)=p_{\text {OUT }}^{(0)}(y, t)$, the reactive "constant" in (1.6) is

$$
\kappa(t)=\frac{P \sqrt{\sigma(0, t)}}{\sqrt{\pi}}
$$


3. Numerical simulations in one dimension. The explicit analytical solution of the FPE (1.3) with the initial condition (1.5) and the radiation boundary condition (1.6) for the case of vanishing drift $(a=0)$ and constant diffusion coefficient $(\sigma(x, t)=\sigma)$ was first given by Bryan in 1891 [36] (see [37, §14.2, p.358])

$$
\begin{aligned}
p\left(x, t \mid x_{0}\right)= & \frac{1}{\sqrt{4 \pi \sigma t}}\left[\exp \left\{-\frac{\left(x-x_{0}\right)^{2}}{4 \sigma t}\right\}+\exp \left\{-\frac{\left(x+x_{0}\right)^{2}}{4 \sigma t}\right\}\right] \\
& -\frac{\kappa}{\sigma} \exp \left\{\frac{\kappa\left(x+x_{0}+\kappa t\right)}{\sigma}\right\} \operatorname{erfc}\left[\frac{x+x_{0}+2 \kappa t}{\sqrt{4 \sigma t}}\right] .
\end{aligned}
$$

The first term in (3.1) is the fundamental solution of (1.3) and (1.5) with a reflecting boundary condition, whereas the second term may be transformed into

$$
-\frac{\kappa}{\sqrt{\pi \sigma^{3} t}} \int_{0}^{\infty} \exp \left\{-\frac{\kappa \xi}{\sigma}\right\} \exp \left\{-\frac{\left(x+x_{0}+\xi\right)^{2}}{4 \sigma t}\right\} d \xi
$$

which represents the density due to a line of exponentially decreasing sinks extending from $-x_{0}$ to $-\infty$. The method of Laplace transforming (1.3) with respect to $t$ was later employed [1], 38, to obtain explicit analytical solution for the FPE (1.3)-(1.5) with constants diffusion coefficient and (not necessarily vanishing) drift term $a(x, t)=a$

$$
\begin{aligned}
& p\left(x, t \mid x_{0}\right)= \\
& \frac{1}{\sqrt{4 \pi \sigma t}}\left[\exp \left\{-\frac{\left(x-x_{0}-a t\right)^{2}}{4 \sigma t}\right\}+\exp \left\{-\frac{a x_{0}}{\sigma}-\frac{\left(x+x_{0}-a t\right)^{2}}{4 \sigma t}\right\}\right] \\
& -\frac{2 \kappa+a}{2 \sigma} \exp \left\{\frac{a x+\kappa\left[x+x_{0}+(\kappa+a) t\right]}{\sigma}\right\} \operatorname{erfc}\left[\frac{x+x_{0}+(2 \kappa+a) t}{\sqrt{4 \sigma t}}\right] .
\end{aligned}
$$

Setting $\kappa=0$ in (3.2) reduces to Smoluchowski's [39] explicit analytical solution for a reflecting boundary with a constant drift term, while setting $a=0$ reduces to Bryan's solution (3.1).

We conducted several numerical experiments in which $n=10^{7}$ trajectories were simulated according to the Euler scheme (1.7) with the boundary behavior (1.10). The diffusion coefficient was constant $\sigma=1$ and the reactive constant was $\kappa=1$, giving $P=\sqrt{\pi}$ in eq.(2.29). The trajectories were initially located at $x_{0}=1$, their statistics were collected at time $t=1$, and compared to the predicted $p\left(x, t=1 \mid x_{0}=1\right)$. The convergence of the scheme was tested by using four different time steps $\Delta t=10^{-1}, 10^{-2}, 10^{-3}, 10^{-4}$.

The first experiment corresponds to a vanishing drift $a=0$. Figure 6.1 shows the convergence of the numerical scheme to the analytic solution (3.1). The rate of convergence of the numerical scheme to the analytic solution is $\sqrt{\Delta t}$. This is demonstrated, for example, by the survival probability

$$
p_{\text {sur }}\left(x_{0}, t\right)=\int_{0}^{\infty} p\left(x, t \mid x_{0}\right) d x
$$

of finding the trajectory inside the domain at time $t$, that is, the probability that the trajectory was not absorbed prior to $t$. Integrating (3.1) gives $p_{\text {sur }}(1,1)=0.77095 \ldots$ for $\sigma=\kappa=1$. The survival probability is estimated numerically by the ratio of the number of survived (unabsorbed) trajectories $n_{\text {sur }}$ and the total number of simulated trajectories $n=$ $10^{7}$. Table 6.1 shows that the convergence rate of the estimated survival probability to its analytic value is $\sqrt{\Delta t}$ as predicted by our boundary layer analysis. The statistical estimation 
(variance) error due to the finite number of simulated trajectories is $\sqrt{p_{\text {sur }}\left(1-p_{\text {sur }}\right) / n}=$ $0.00013 \ldots$, which is an order of magnitude smaller than the smallest (bias) error obtained for $\Delta t=10^{-4}$ (see Table 6.1).

In the second experiment the drift term $a=-1$ shifts the density leftwards, and causes more trajectories to react with the boundary. Figure 6.2 shows the convergence of the numerical scheme to the analytic solution (3.2).

The final experiment corresponds to a reflecting boundary, $P=\kappa=0$ and a constant non-vanishing drift towards the boundary $a=-1$. We simulated $n=10^{8}$ trajectories to obtain a finer resolution at the boundary. Figure 6.3 shows a comparison between the analytical solution (3.2) and the numerical densities for $\Delta t=10^{-1}, 10^{-2}$. The no flux condition $J=0$ of a reflecting boundary together with (1.4) gives a negative boundary derivative $p_{y}(0, t)=-p(0, t)<0$. In particular, the analytic solution (3.2) satisfies $p_{y}(0,1)=$ $-p(0,1)=-(2+\sqrt{\pi}) /(2 \sqrt{\pi}) \approx-1.06$. The numerical densities, however, are flat at the boundary. Their first derivatives vanish at the boundary, as predicted in (2.2) and shown in Figure 6.3. The first derivative changes from 0 to $O(1)$ on an interval of length $O(\sqrt{\Delta t})$, manifesting a boundary layer behavior, though there is no such behavior in the density itself.

4. Diffusion in $\mathbb{R}^{d}$ with partial oblique reflection at the boundary. We consider the $d$-dimensional stochastic dynamics

$$
\dot{\boldsymbol{x}}=\boldsymbol{a}(\boldsymbol{x}, t)+\sqrt{2} \boldsymbol{B}(t) \dot{\boldsymbol{w}}
$$

in the half space

$$
\Omega=\left\{\boldsymbol{x}=\left(x_{1}, x_{2}, \ldots, x_{d}\right) \in \mathbb{R}^{d}: x_{1}>0\right\}
$$

where $\boldsymbol{w}$ is a vector of $d$ independent Brownian motions and we assume that the diffusion tensor $\boldsymbol{\sigma}(t)=\boldsymbol{B}(t) \boldsymbol{B}^{T}(t)$ is uniformly positive definite for all $t \geq s$. The case of spacedependent diffusion involves many technically complicated calculations and will be considered in a separate paper. We use henceforth the abbreviation $\boldsymbol{\sigma}(t)=\boldsymbol{\sigma}$. The radiation condition (1.6) becomes

$$
-\boldsymbol{J}(\boldsymbol{y}, t \mid \boldsymbol{x}, s) \cdot \boldsymbol{n}=\kappa(\boldsymbol{y}, t) p(\boldsymbol{y}, t \mid \boldsymbol{x}, s), \quad \text { for } \quad \boldsymbol{y} \in \partial \Omega, \boldsymbol{x} \in \Omega,
$$

where the components of the flux vector $\boldsymbol{J}(\boldsymbol{y}, t \mid \boldsymbol{x}, s)$ are defined by

$$
J^{k}(\boldsymbol{y}, t \mid \boldsymbol{x}, s)=-\left[a^{k}(\boldsymbol{y}, t) p(\boldsymbol{y}, t \mid \boldsymbol{x}, s)\right]+\sum_{j=1}^{d} \frac{\partial}{\partial y_{j}}\left[\sigma^{j, k} p(\boldsymbol{y}, t \mid x, s)\right],
$$

where $\sigma^{j, k}$ are the elements of the diffusion matrix $\boldsymbol{\sigma}$. The Fokker-Plank equation for the pdf of $\boldsymbol{x}(t)$ can be written as

$$
\frac{\partial p(\boldsymbol{y}, t \mid \boldsymbol{x}, s)}{\partial t}=-\nabla \boldsymbol{y} \cdot \boldsymbol{J}(\boldsymbol{y}, t \mid \boldsymbol{x}, s) \quad \text { for all } \quad \boldsymbol{y}, \boldsymbol{x} \in \Omega .
$$

If $\boldsymbol{x} \in \Omega$, but

$$
\boldsymbol{x}^{\prime}=\boldsymbol{x}+\boldsymbol{a}(\boldsymbol{x}, t) \Delta t+\sqrt{2} \boldsymbol{B}(t) \Delta \boldsymbol{w}(t, \Delta t) \notin \Omega,
$$

the Euler scheme for (4.1) with oblique reflection in $\partial \Omega$ reflects the point $\boldsymbol{x}^{\prime}$ obliquely in the constant direction of $\boldsymbol{v}$ to a point $\boldsymbol{x}^{\prime \prime} \in \Omega$, as described below. First, we denote by $\boldsymbol{x}_{B}^{\prime}$ the normal projection of a point $\boldsymbol{x}^{\prime}$ on $\partial \Omega$, that is, $\boldsymbol{x}_{B}^{\prime}=\boldsymbol{x}^{\prime}-\left(\boldsymbol{x}^{\prime} \cdot \boldsymbol{n}\right) \boldsymbol{n}$. Then we write the Euler scheme for (4.1) with partially reflecting boundary as

$$
\boldsymbol{x}(t+\Delta t)=\left\{\begin{array}{l}
\boldsymbol{x}^{\prime} \text { for } \boldsymbol{x}^{\prime} \in \Omega \\
\boldsymbol{x}^{\prime \prime} \quad \text { w.p. } 1-P\left(\boldsymbol{x}_{B}^{\prime}\right) \sqrt{\Delta t}, \quad \text { if } \quad \boldsymbol{x}^{\prime} \notin \Omega, \\
\text { terminate trajectory w.p. } P\left(\boldsymbol{x}_{B}^{\prime}\right) \sqrt{\Delta t}, \quad \text { if } \quad \boldsymbol{x}^{\prime} \notin \Omega .
\end{array}\right.
$$


The value of the termination probability $P\left(\boldsymbol{x}_{B}^{\prime}\right) \sqrt{\Delta t}$, that varies continuously in the boundary, is evaluated at the normal projection of the point $\boldsymbol{x}^{\prime}$ on the boundary. The oblique reflection in the direction of the unit vector $\boldsymbol{v}\left(v_{1} \neq 0\right)$ is defined by

$$
\boldsymbol{x}^{\prime \prime}=\boldsymbol{x}^{\prime}-\frac{2 x_{1}^{\prime}}{v_{1}} \boldsymbol{v}
$$

Note that $x_{1}^{\prime \prime}=-x_{1}^{\prime}$ guarantees that the reflected point of a crossing trajectory is inside the domain $\Omega$. The fact that the normal components of $\boldsymbol{x}^{\prime \prime}$ and $\boldsymbol{x}^{\prime}$ are of equal lengths makes the high-dimensional boundary layer analysis similar to that in one dimension. Normal reflection corresponds to $\boldsymbol{v}=\boldsymbol{n}=(1,0, \ldots, 0)$.

We note that for a point $\boldsymbol{y} \in \Omega$, we can write $\operatorname{Pr}\left\{\boldsymbol{x}^{\prime \prime}=\boldsymbol{y}\right\}=\operatorname{Pr}\left\{\boldsymbol{x}^{\prime}=\boldsymbol{y}^{\prime}\right\}$, where

$$
\boldsymbol{y}=\boldsymbol{y}^{\prime}-\frac{2 \boldsymbol{y}^{\prime} \cdot \boldsymbol{n}}{v_{1}} \boldsymbol{v}
$$

is the oblique reflection of $\boldsymbol{y}^{\prime}$ (see fig. 6.4). Given $\boldsymbol{y}$, equation (4.7) defines $\boldsymbol{y}^{\prime}$ as

$$
\boldsymbol{y}^{\prime}=\boldsymbol{y}-2 \frac{y_{1}}{v_{1}} \boldsymbol{v}
$$

As in the one-dimensional case, the forward Kolmogorov equation is

$$
\begin{aligned}
p_{\Delta t}(\boldsymbol{y}, t+\Delta t)= & \int_{x_{1}>0} \frac{p_{\Delta t}(\boldsymbol{x}, t)}{(4 \pi \Delta t)^{d / 2} \sqrt{\operatorname{det} \boldsymbol{\sigma}}}\left\{\exp \left[-\frac{\mathcal{B}(\boldsymbol{x}+\boldsymbol{a}(\boldsymbol{x}, t) \Delta t, \boldsymbol{y})}{4 \Delta t}\right]+\right. \\
& \left.\left(1-P\left(\boldsymbol{y}_{B}^{\prime}\right) \sqrt{\Delta t}\right) \exp \left[-\frac{\mathcal{B}\left(\boldsymbol{x}+\boldsymbol{a}(\boldsymbol{x}, t) \Delta t, \boldsymbol{y}^{\prime}\right)}{4 \Delta t}\right]\right\} d \boldsymbol{x},
\end{aligned}
$$

where

$$
\mathcal{B}(\boldsymbol{x}, \boldsymbol{y})=(\boldsymbol{x}-\boldsymbol{y})^{T} \boldsymbol{\sigma}^{-1}(\boldsymbol{x}-\boldsymbol{y}) .
$$

We construct a boundary layer of width $O(\sqrt{\Delta t})$ in the normal direction to the boundary. The layer extends infinitely in the $d-1$ directions tangent to the boundary

$$
p_{B L}\left(\eta_{1}, y_{2}, \ldots, y_{d}, t\right)=p_{\Delta t}\left(\eta_{1} \sqrt{\Delta t}, y_{2}, \ldots, y_{d}, t\right) .
$$

In other words, $p_{B L}\left(\eta_{1} \boldsymbol{n}+\boldsymbol{y}_{B}, t\right)=p_{\Delta t}\left(\eta_{1} \sqrt{\Delta t} \boldsymbol{n}+\boldsymbol{y}_{B}, t\right)$, where $\boldsymbol{y}_{B}=\left(0, y_{2}, y_{3}, \ldots, y_{d}\right)$. As in the one-dimensional case, we assume the asymptotic expansion

$$
p_{B L}\left(\eta_{1} \boldsymbol{n}+\boldsymbol{y}_{B}, t\right) \sim p_{B L}^{(0)}\left(\eta_{1} \boldsymbol{n}+\boldsymbol{y}_{B}, t\right)+\sqrt{\Delta t} p_{B L}^{(1)}\left(\eta_{1} \boldsymbol{n}+\boldsymbol{y}_{B}, t\right)+\ldots
$$

and substitute

$$
\boldsymbol{x}=\boldsymbol{y}_{B}+\sqrt{\Delta t} \boldsymbol{\xi}
$$

in the integral (4.9). We obtain

$$
\begin{aligned}
& p_{B L}\left(\eta_{1} \boldsymbol{n}+\boldsymbol{y}_{B}, t+\Delta t\right)=\int_{\xi_{1}>0} \frac{p_{B L}\left(\xi_{1} \boldsymbol{n}+\boldsymbol{y}_{B}+\sqrt{\Delta t} \boldsymbol{\xi}_{B}, t\right)}{(4 \pi)^{d / 2} \sqrt{\operatorname{det} \boldsymbol{\sigma}}} \times \\
& \left\{\exp \left[-\frac{\mathcal{B}\left(\boldsymbol{\xi}+\boldsymbol{a}\left(\boldsymbol{y}_{B}, t\right) \sqrt{\Delta t}, \eta_{1} \boldsymbol{n}\right)}{4}\right]+\left(1-P\left(\boldsymbol{y}_{B}^{\prime}\right) \sqrt{\Delta t}\right) \times\right. \\
& \left.\exp \left[-\frac{1}{4} \mathcal{B}\left(\boldsymbol{\xi}+\boldsymbol{a}\left(\boldsymbol{y}_{B}, t\right) \sqrt{\Delta t}, \eta_{1} \boldsymbol{n}-\frac{2 \eta_{1}}{v_{1}} \boldsymbol{v}\right)\right]\right\} d \boldsymbol{\xi}+O(\Delta t) .
\end{aligned}
$$


We calculate separately the integral of the first and second terms in the braces. Substituting in the first integral

$$
\boldsymbol{z}=\boldsymbol{\sigma}^{-1 / 2}\left(\boldsymbol{\xi}-\eta_{1} \boldsymbol{n}\right)
$$

transforms the domain of integration to

$$
\boldsymbol{z} \cdot \tilde{\boldsymbol{n}}>-\frac{\eta_{1}}{\sqrt{\sigma_{n}}}
$$

where $\tilde{\boldsymbol{n}}=\frac{\boldsymbol{\sigma}^{1 / 2} \boldsymbol{n}}{\left\|\boldsymbol{\sigma}^{1 / 2} \boldsymbol{n}\right\|}$ is a unit vector, and $\sigma_{n}=\boldsymbol{n}^{T} \boldsymbol{\sigma} \boldsymbol{n}=\left\|\boldsymbol{\sigma}^{1 / 2} \boldsymbol{n}\right\|^{2}$. Similarly, we transform the second integral by substituting $\boldsymbol{z}^{\prime}=\boldsymbol{\sigma}^{-1 / 2}\left(\boldsymbol{\xi}-\eta_{1} \boldsymbol{n}+\frac{2 \eta_{1}}{v_{1}} \boldsymbol{v}\right)$. Using the expansion (4.12), we obtain at the leading order the integral equation

$$
\begin{aligned}
& p_{B L}^{(0)}\left(\eta_{1} \boldsymbol{n}+\boldsymbol{y}_{B}, t\right)= \\
& \frac{1}{(4 \pi)^{d / 2}} \int_{\boldsymbol{z} \cdot \tilde{\boldsymbol{n}}>-\frac{\eta_{1}}{\sqrt{\sigma_{n}}}} p_{B L}^{(0)}\left(\left(\eta_{1}+\sqrt{\sigma_{n}} \boldsymbol{z} \cdot \tilde{\boldsymbol{n}}\right) \boldsymbol{n}+\boldsymbol{y}_{B}, t\right) \exp \left[-\frac{\|\boldsymbol{z}\|^{2}}{4}\right] d \boldsymbol{z}+ \\
& \frac{1}{(4 \pi)^{d / 2}} \int_{\boldsymbol{z}^{\prime} \cdot \tilde{\boldsymbol{n}}>\frac{\eta_{1}}{\sqrt{\sigma_{n}}}} p_{B L}^{(0)}\left(\left(-\eta_{1}+\sqrt{\sigma_{n}} \boldsymbol{z}^{\prime} \cdot \tilde{\boldsymbol{n}}\right) \boldsymbol{n}+\boldsymbol{y}_{B}, t\right) \exp \left[-\frac{\left\|\boldsymbol{z}^{\prime}\right\|^{2}}{4}\right] d \boldsymbol{z}^{\prime} .
\end{aligned}
$$

Integrating in the $d-1$ directions orthogonal to $\tilde{\boldsymbol{n}}$, yields

$$
\begin{aligned}
& p_{B L}^{(0)}\left(\eta_{1} \boldsymbol{n}+\boldsymbol{y}_{B}, t\right)=\frac{1}{\sqrt{4 \pi}} \int_{-\frac{\eta_{1}}{\sqrt{\sigma_{n}}}}^{\infty} p_{B L}^{(0)}\left(\left(\eta_{1}+\sqrt{\sigma_{n}} u\right) \boldsymbol{n}+\boldsymbol{y}_{B}, t\right) \exp \left[-\frac{u^{2}}{4}\right] d u+ \\
& \frac{1}{\sqrt{4 \pi}} \int_{\frac{\eta_{1}}{\sqrt{\sigma_{n}}}}^{\infty} p_{B L}^{(0)}\left(\left(-\eta_{1}+\sqrt{\sigma_{n}} u\right) \boldsymbol{n}+\boldsymbol{y}_{B}, t\right) \exp \left[-\frac{u^{2}}{4}\right] d u= \\
& \frac{1}{\sqrt{4 \pi \sigma_{n}}} \int_{0}^{\infty} p_{B L}^{(0)}\left(u \boldsymbol{n}+\boldsymbol{y}_{B}, t\right)\left\{\exp \left[-\frac{\left(u-\eta_{1}\right)^{2}}{4 \sigma_{n}}\right]+\exp \left[-\frac{\left(u+\eta_{1}\right)^{2}}{4 \sigma_{n}}\right]\right\} d u
\end{aligned}
$$

This is the same leading order integral equation as that of the one-dimensional case (2.9), so the solution is independent of $\eta_{1}$, and matching to the outer solution gives

$$
p_{B L}^{(0)}\left(\eta_{1} \boldsymbol{n}+\boldsymbol{y}_{B}, t\right)=p_{O U T}^{(0)}\left(\boldsymbol{y}_{B}, t\right) .
$$

To evaluate the $O(\sqrt{\Delta t})$ terms, we expand in the first integral in (4.14)

$$
\begin{aligned}
\mathcal{B}\left(\boldsymbol{\xi}+\boldsymbol{a}\left(\boldsymbol{y}_{B}, t\right) \sqrt{\Delta t}, \eta_{1} \boldsymbol{n}\right)= & \left(\boldsymbol{\xi}-\eta_{1} \boldsymbol{n}\right) \cdot \boldsymbol{\sigma}^{-1}\left(\boldsymbol{\xi}-\eta_{1} \boldsymbol{n}\right)+ \\
& \sqrt{\Delta t} 2 \boldsymbol{a}\left(\boldsymbol{y}_{B}, t\right) \cdot \boldsymbol{\sigma}^{-1}\left(\boldsymbol{\xi}-\eta_{1} \boldsymbol{n}\right) .
\end{aligned}
$$

and in the second integral

$$
\begin{aligned}
\mathcal{B}\left(\boldsymbol{\xi}+\boldsymbol{a}\left(\boldsymbol{y}_{B}, t\right) \sqrt{\Delta t}, \eta_{1} \boldsymbol{n}-\frac{2 \eta_{1}}{v_{1}} \boldsymbol{v}\right)= & \left(\boldsymbol{\xi}-\eta_{1} \boldsymbol{n}+\frac{2 \eta_{1}}{v_{1}} \boldsymbol{v}\right) \cdot \boldsymbol{\sigma}^{-1}\left(\boldsymbol{\xi}-\eta_{1} \boldsymbol{n} \frac{2 \eta_{1}}{v_{1}} \boldsymbol{v}\right)+ \\
& \sqrt{\Delta t} 2 \boldsymbol{a}\left(\boldsymbol{y}_{B}, t\right) \cdot \boldsymbol{\sigma}^{-1}\left(\boldsymbol{\xi}-\eta_{1} \boldsymbol{n} \frac{2 \eta_{1}}{v_{1}} \boldsymbol{v}\right)
\end{aligned}
$$


The $O(\sqrt{\Delta t})$ contribution of the drift term for the first exponential term is

$$
\begin{aligned}
& -\frac{1}{4} \int_{\xi_{1}>0} \frac{p_{\text {OUT }}^{(0)}\left(\boldsymbol{y}_{B}, t\right)}{(4 \pi)^{d / 2} \sqrt{\operatorname{det} \boldsymbol{\sigma}}} \exp \left\{-\frac{\mathcal{B}\left(\boldsymbol{\xi}, \eta_{1} \boldsymbol{n}\right)}{4}\right\}\left[2 \boldsymbol{a}\left(\boldsymbol{y}_{B}, t\right) \cdot \boldsymbol{\sigma}^{-1}\left(\boldsymbol{\xi}-\eta_{1} \boldsymbol{n}\right)\right] d \boldsymbol{\xi}= \\
& -\frac{1}{4} \frac{p_{O U T}^{(0)}\left(\boldsymbol{y}_{B}, t\right)}{\sqrt{4 \pi}} 2 \boldsymbol{a}\left(\boldsymbol{y}_{B}, t\right) \cdot \boldsymbol{\sigma}^{-1 / 2} \tilde{\boldsymbol{n}} \int_{-\eta_{1} / \sqrt{\sigma_{n}}}^{\infty} u e^{-u^{2} / 4} d u= \\
& -\frac{1}{2} \frac{p_{O U T}^{(0)}\left(\boldsymbol{y}_{B}, t\right)}{\sqrt{\pi \sigma_{n}}} \boldsymbol{a}\left(\boldsymbol{y}_{B}, t\right) \cdot \boldsymbol{n} \exp \left\{\frac{-\eta_{1}^{2}}{4 \sigma_{n}}\right\} .
\end{aligned}
$$

The second exponential has the same contribution, so the overall contribution of the drift to the $O(\sqrt{\Delta t})$ term is

$$
-\frac{p_{O U T}^{(0)}\left(\boldsymbol{y}_{B}, t\right)}{\sqrt{\pi \sigma_{n}}} \boldsymbol{a}\left(\boldsymbol{y}_{B}, t\right) \cdot \boldsymbol{n} \exp \left\{\frac{-\eta_{1}^{2}}{4 \sigma_{n}}\right\}
$$

Now, we expand

$$
\begin{aligned}
& p_{B L}^{(0)}\left(\left(\eta_{1}+\sqrt{\sigma_{n}} \boldsymbol{z} \cdot \tilde{\boldsymbol{n}}\right) \boldsymbol{n}+\boldsymbol{y}_{B}+\sqrt{\Delta t}\left(\boldsymbol{\sigma}^{1 / 2} \boldsymbol{z}\right)_{B}, t\right)=p_{B L}^{(0)}\left(\left(\eta_{1}+\sqrt{\sigma_{n}} \boldsymbol{z} \cdot \tilde{\boldsymbol{n}}\right) \boldsymbol{n}+\boldsymbol{y}_{B}, t\right)+ \\
& \sqrt{\Delta t} \nabla p_{B L}^{(0)}\left(\left(\eta_{1}+\sqrt{\sigma_{n}} \boldsymbol{z} \cdot \tilde{\boldsymbol{n}}\right) \boldsymbol{n}+\boldsymbol{y}_{B}, t\right) \cdot\left(\boldsymbol{\sigma}^{1 / 2} \boldsymbol{z}\right)_{B}+O(\Delta t) .
\end{aligned}
$$

Together with (4.17), the expansion (4.22) reduces to

$$
\begin{aligned}
& p_{B L}^{(0)}\left(\left(\eta_{1}+\sqrt{\sigma_{n}} \boldsymbol{z} \cdot \tilde{\boldsymbol{n}}\right) \boldsymbol{n}+\boldsymbol{y}_{B}+\sqrt{\Delta t}\left(\boldsymbol{\sigma}^{1 / 2} \boldsymbol{z}\right)_{B}, t\right)= \\
& p_{O U T}^{(0)}\left(\boldsymbol{y}_{B}, t\right)+\sqrt{\Delta t} \nabla p_{O U T}^{(0)}\left(\boldsymbol{y}_{B}, t\right) \cdot\left(\boldsymbol{\sigma}^{1 / 2} \boldsymbol{z}\right)_{B}+O(\Delta t) .
\end{aligned}
$$

Integrating as above, we obtain the $O(\sqrt{\Delta t})$ integral equation as

$$
\begin{aligned}
& p_{B L}^{(1)}\left(\eta_{1} \boldsymbol{n}+\boldsymbol{y}_{B}, t\right)= \\
& \frac{1}{\sqrt{4 \pi \sigma_{n}}} \int_{0}^{\infty} p_{B L}^{(1)}\left(u \boldsymbol{n}+\boldsymbol{y}_{B}, t\right)\left\{\exp \left[-\frac{\left(u-\eta_{1}\right)^{2}}{4 \sigma_{n}}\right]+\exp \left[-\frac{\left(u+\eta_{1}\right)^{2}}{4 \sigma_{n}}\right]\right\} d u- \\
& \frac{P\left(\boldsymbol{y}_{B}^{\prime}\right) p_{O U T}^{(0)}\left(\boldsymbol{y}_{B}, t\right)}{\sqrt{4 \pi \sigma_{n}}} \int_{0}^{\infty} \exp \left[-\frac{\left(u+\eta_{1}\right)^{2}}{4 \sigma_{n}}\right] d u+ \\
& \frac{1}{\sqrt{4 \pi}} \int_{\frac{\eta_{1}}{\sqrt{\sigma_{n}}}}^{\infty} \nabla p_{O U T}^{(0)}\left(\boldsymbol{y}_{B}, t\right) \cdot\left(2 \boldsymbol{\sigma}^{1 / 2} u \tilde{\boldsymbol{n}}-\frac{2 \eta_{1}}{v_{1}} \boldsymbol{v}\right)_{B} \exp \left[-\frac{u^{2}}{4}\right] d u- \\
& \frac{p_{O U T}^{(0)}\left(\boldsymbol{y}_{B}, t\right)}{\sqrt{\pi \sigma_{n}}} \boldsymbol{a}\left(\boldsymbol{y}_{B}, t\right) \cdot \boldsymbol{n} \exp \left\{\frac{-\eta_{1}^{2}}{4 \sigma_{n}}\right\} .
\end{aligned}
$$

Differentiating with respect to $\eta_{1}$ and integrating by parts (as was done in the one dimen- 
sional case), we arrive at the integral equation

$$
\begin{aligned}
& \frac{\partial p_{B L}^{(1)}\left(\eta_{1} \boldsymbol{n}+\boldsymbol{y}_{B}, t\right)}{\partial n}= \\
& \frac{1}{\sqrt{4 \pi \sigma_{n}}} \int_{0}^{\infty} \frac{\partial p_{B L}^{(1)}\left(u \boldsymbol{n}+\boldsymbol{y}_{B}, t\right)}{\partial n}\left\{\exp \left[-\frac{\left(u-\eta_{1}\right)^{2}}{4 \sigma_{n}}\right]-\exp \left[-\frac{\left(u+\eta_{1}\right)^{2}}{4 \sigma_{n}}\right]\right\} d u- \\
& \frac{P\left(\boldsymbol{y}_{B}^{\prime}\right) p_{O U T}^{(0)}\left(\boldsymbol{y}_{B}, t\right)}{\sqrt{4 \pi \sigma_{n}}} \exp \left[\frac{-\eta_{1}^{2}}{4 \sigma_{n}}\right]+ \\
& \nabla p_{O U T}^{(0)}\left(\boldsymbol{y}_{B}, t\right) \cdot\left\{-\frac{1}{\sqrt{\pi \sigma_{n}}}\left[\frac{\boldsymbol{\sigma} \boldsymbol{n}}{\sigma_{n}}-\frac{\boldsymbol{v}}{v_{1}}\right] \eta_{1} \exp \left[\frac{-\eta_{1}^{2}}{4 \sigma_{n}}\right]-\boldsymbol{v} \frac{\operatorname{erfc}\left(\frac{\eta_{1}}{2 \sqrt{\sigma_{n}}}\right)}{v_{1}}\right\}_{B}+ \\
& \frac{p_{O U T}^{(0)}\left(\boldsymbol{y}_{B}, t\right)}{\sqrt{\pi \sigma_{n}}} \boldsymbol{a}\left(\boldsymbol{y}_{B}, t\right) \cdot \boldsymbol{n} \frac{\eta_{1}}{2 \sigma_{n}} \exp \left[\frac{-\eta_{1}^{2}}{4 \sigma_{n}}\right] .
\end{aligned}
$$

The Wiener-Hopf method requires the extension of the erfc function discontinuously as an odd function, that is, to define $\widehat{\operatorname{erfc}}(x)=\operatorname{sgn}(x) \operatorname{erfc}(|x|)$. Following the calculations of the one dimensional case, it remains to determine the small $k$ behavior of the Fourier transform of $\widetilde{\operatorname{erfc}}(x)$. Using

$$
\int_{-\infty}^{\infty} \widetilde{\operatorname{erfc}}\left(\frac{\eta}{2 \sqrt{\sigma_{n}}}\right) \exp \{i k \eta\} d \eta \sim 2 i k \int_{0}^{\infty} \operatorname{erfc}\left(\frac{\eta}{2 \sqrt{\sigma_{n}}}\right) \eta d \eta=2 i k \sigma_{n}
$$

we obtain, as in (2.24),

$$
\begin{aligned}
\hat{\phi}(k) \sim & 2 i k\left\{\frac{P\left(\boldsymbol{y}_{B}^{\prime}\right) p_{O U T}^{(0)}\left(\boldsymbol{y}_{B}, t\right) \sqrt{\sigma_{n}}}{\sqrt{\pi}}-2 \sigma_{n} \nabla p_{O U T}^{(0)}\left(\boldsymbol{y}_{B}, t\right) \cdot\left[\frac{\boldsymbol{\sigma n}}{\sigma_{n}}-\frac{\boldsymbol{v}}{2 v_{1}}\right]_{B}+\right. \\
& \left.p_{O U T}^{(0)}\left(\boldsymbol{y}_{B}, t\right) \boldsymbol{a}\left(\boldsymbol{y}_{B}, t\right) \cdot \boldsymbol{n}\right\} \quad \text { as } \quad k \rightarrow 0 .
\end{aligned}
$$

Therefore,

$$
\begin{aligned}
& \lim _{\eta_{1} \rightarrow \infty} \frac{\partial p_{B L}^{(1)}\left(\eta_{1} \boldsymbol{n}+\boldsymbol{y}_{B}, t\right)}{\partial n}= \\
& \left\{\frac{P\left(\boldsymbol{y}_{B}^{\prime}\right) p_{O U T}^{(0)}\left(\boldsymbol{y}_{B}, t\right)}{\sqrt{\pi \sigma_{n}}}-2 \nabla p_{O U T}^{(0)}\left(\boldsymbol{y}_{B}, t\right) \cdot\left[\frac{\boldsymbol{\sigma} \boldsymbol{n}}{\sigma_{n}}-\frac{\boldsymbol{v}}{2 v_{1}}\right]_{B}+p_{O U T}^{(0)}\left(\boldsymbol{y}_{B}, t\right) \frac{\boldsymbol{a}\left(\boldsymbol{y}_{B}, t\right) \cdot \boldsymbol{n}}{\sigma_{n}}\right\} .
\end{aligned}
$$

Combining with the matching condition

$$
\lim _{\eta \rightarrow \infty} \frac{\partial p_{B L}^{(1)}\left(\eta_{1} \boldsymbol{n}+\boldsymbol{y}_{B}, t\right)}{\partial n}=\frac{\partial p_{O U T}^{(0)}\left(\boldsymbol{y}_{B}, t\right)}{\partial n},
$$

we obtain

$$
\begin{aligned}
& \frac{\partial p_{O U T}^{(0)}\left(\boldsymbol{y}_{B}, t\right)}{\partial n}= \\
& \left\{\frac{P\left(\boldsymbol{y}_{B}\right) p_{O U T}^{(0)}\left(\boldsymbol{y}_{B}, t\right)}{\sqrt{\pi \sigma_{n}}}-2 \nabla p_{O U T}^{(0)}\left(\boldsymbol{y}_{B}, t\right) \cdot\left[\frac{\boldsymbol{\sigma} \boldsymbol{n}}{\sigma_{n}}-\frac{\boldsymbol{v}}{2 v_{1}}\right]_{B}+p_{O U T}^{(0)}\left(\boldsymbol{y}_{B}, t\right) \frac{\boldsymbol{a}\left(\boldsymbol{y}_{B}, t\right) \cdot \boldsymbol{n}}{\sigma_{n}}\right\} .
\end{aligned}
$$


The requirement that the pdf of the limiting diffusion process satisfies the Robin boundary condition leads to the only possible choice

$$
v=\frac{\sigma n}{\|\sigma n\|} .
$$

Otherwise, we obtain an oblique derivative boundary condition. Since $\boldsymbol{y}_{B}^{\prime} \rightarrow \boldsymbol{y}_{B}$ as $\Delta t \rightarrow 0$, we obtain the Robin boundary condition

$$
\begin{aligned}
-\boldsymbol{J}_{O U T}\left(\boldsymbol{y}_{B}, t\right) \cdot \boldsymbol{n} & =\nabla p_{O U T}^{(0)}\left(\boldsymbol{y}_{B}, t\right) \cdot \boldsymbol{\sigma n}-p_{O U T}^{(0)}\left(\boldsymbol{y}_{B}, t\right) \boldsymbol{a}\left(\boldsymbol{y}_{B}, t\right) \cdot \boldsymbol{n} \\
& =\frac{P\left(\boldsymbol{y}_{B}\right) p_{O U T}^{(0)}\left(\boldsymbol{y}_{B}, t\right) \sqrt{\sigma_{n}}}{\sqrt{\pi}} .
\end{aligned}
$$

The reflection direction $\boldsymbol{v}$ of crossing trajectories is the co-normal direction $\boldsymbol{\sigma n}$. Normal reflection (i.e., replacing $\boldsymbol{v}$ by $\boldsymbol{n}$ ) gives rise to the boundary normal flux if and only if $\boldsymbol{n}$ is an eigenvector of the diffusion tensor $\boldsymbol{\sigma}$. The limit of the outer solution as $\Delta t \rightarrow 0$ is the solution of the Fokker-Planck equation (4.4) with the radiation boundary condition

$$
-\boldsymbol{J}(\boldsymbol{y}, t) \cdot \boldsymbol{n}=\kappa(\boldsymbol{y}) p(\boldsymbol{y}, t) \quad \text { for } \quad \boldsymbol{y} \in \partial \Omega,
$$

where the reactive "constant" is

$$
\kappa(\boldsymbol{y})=\frac{P(\boldsymbol{y}) \sqrt{\sigma_{n}}}{\sqrt{\pi}} .
$$

Note that normal reflection will not recover the normal flux of the radiation condition if $\boldsymbol{n}$ is not an eigenvector of $\boldsymbol{\sigma}$.

5. Numerical simulations in two dimensions. To illustrate the co-normal reflection law (4.25) in the Euler scheme (4.5)-(4.7) in the half plane $x \geq 0$, we ran several numerical experiments. The simulations show the convergence of the pdf of the numerical solution to that of the FPE with the radiation boundary condition (4.26)-(4.27). Unlike the one-dimensional case, no explicit solution of the anisotropic Robin problem for the FPE in the half plane is available, so we compare the statistics of the simulated trajectories with a numerical solution of the FPE. The latter is constructed by the stable Crank-Nicolson scheme on lattice points, where in each time step the sparse linear system is solved by the conjugate gradient method.

In all numerical experiments the initial point is $\left(x_{0}, y_{0}\right)=(0.3,0)$ and the statistics is collected at time $T=0.5$. We choose the reactive constant $\kappa=1$ and the diffusion matrix $\boldsymbol{B}$ in (4.1)

$$
\boldsymbol{B}=\left(\begin{array}{cc}
0.3 & 0.4 \\
0 & 1
\end{array}\right)
$$

which gives the anisotropic diffusion tensor

$$
\boldsymbol{\sigma}=\boldsymbol{B} \boldsymbol{B}^{T}=\left(\begin{array}{cc}
0.25 & 0.4 \\
0.4 & 1
\end{array}\right)
$$

We simulate $n=10^{7}$ trajectories with time steps $\Delta t=10^{-1}, 10^{-2}, 10^{-3}, 10^{-4}$ in each experiment.

In the first experiment the drift vanishes, $\boldsymbol{a}=0$. The normal $\boldsymbol{n}=(1,0)$ and the co-normal $\boldsymbol{\sigma} \boldsymbol{n}=(0.25,0.4)$ point in different directions. The simulated trajectories are reflected in the co-normal direction according to the prescription (4.25). The simulated and 
the numerical solution of the FPE give the marginal densities shown in Figures 6.5 and 6.6 Figure 6.5 shows the marginal density of $x(T)$,

$$
p\left(x, T \mid x_{0}, y_{0}\right)=\int_{-\infty}^{\infty} p\left(x, y, T \mid x_{0}, y_{0}\right) d y,
$$

while Figure 6.6 shows the marginal density of $y(T)$,

$$
p\left(y, T \mid x_{0}, y_{0}\right)=\int_{0}^{\infty} p\left(x, y, T \mid x_{0}, y_{0}\right) d x .
$$

Table 6.2 gives the computed survival probability and indicates the convergence rate.

We illustrate the importance of using the correct reflection law in the second experiment, in which the simulated trajectories are reflected in the normal direction $\boldsymbol{n}=(1,0)$. Clearly, the marginal density of $x(T)$ coincides with that of the first experiment, because both oblique and normal reflections have the same $x$-coordinate (see (4.6)). However, the plot of the marginal density of $y(T)$ differs significantly from that in the previous experiment. It is apparent from the comparison to the numerical solution of the FPE that the simulation does not recover the Robin boundary condition in the limit $\Delta t \rightarrow 0$ (see Figure 6.7). Note that the peak of the density is at $y>0$, though the reflection is normal. This is due to the anisotropy of the diffusion tensor, which causes the probability flux density vector to have a positive $y$ component.

In the third experiment the drift is the constant vector $\boldsymbol{a}=(-1,0)$ and the diffusion tensor is as in the first experiment. The density is shifted toward the boundary (see Figure 6.8 and Figure 6.9. . The results are summarized in Table 6.3.

6. Summary and Discussion. We have defined a diffusion process with partially reflecting boundary as a limit of Markovian jump processes generated by the Euler scheme for the dynamics in a half space with partial absorption of exiting trajectories and partial oblique reflection in the boundary. We derived an expression for the radiation constant in the Robin boundary condition for the one-dimensional Fokker-Planck equation for the case of diffusion with variable drift and diffusion coefficients, as a function of the absorption probability. We found that the Euler scheme for a diffusion in a half space with variable drift and constant anisotropic diffusion has to be reflected in a particular oblique direction in order to recover the Robin boundary condition. Also for this case we found the radiation "constant" as a function of the local absorption probability on the boundary. We found a boundary layer of width $O(\sqrt{\Delta t})$ in the pdf of the Euler scheme and solved the boundary layer equation, which is of Wiener-Hopf type.

The boundary layer of $p_{\Delta t}(y, t)$ makes the calculation of the boundary flux non-trivial. The net boundary flux of the simulation profile $p_{\Delta t}(y, t)$ is

$$
-J_{\Delta t}(0, t)=\lim _{\Delta t \rightarrow 0} \frac{1}{\Delta t} \frac{P \sqrt{\Delta t}}{\sqrt{4 \pi \sigma \Delta t}} \int_{-\infty}^{0} d y \int_{0}^{\infty} p_{\Delta t}(x, t) \exp \left\{-\frac{(x-y)^{2}}{4 \pi \sigma \Delta t}\right\} d x,
$$

which is the probability of the trajectories that propagate per unit time out of the domain, discounted by the probability of trajectories returned into the domain by the partially reflecting Euler scheme. Changing the order of integration and then changing the variable of integration into $z=x / 2 \sqrt{\sigma \Delta t}$ gives

$$
-J_{\Delta t}(0, t)=P \sqrt{\sigma} \int_{0}^{\infty} \operatorname{erfc}(z) p_{\Delta t}(2 z \sqrt{\sigma \Delta t}, t) d z=\frac{P \sqrt{\sigma}}{\sqrt{\pi}} p_{B L}^{(0)}(0, t)+O(\sqrt{\Delta t}) .
$$

This straightforward calculation of the flux gives the correct radiation constant, provided that

$$
p_{B L}^{(0)}(0, t)=p_{O U T}^{(0)}(0, t) .
$$


The latter, however, depends on the mode of reflecting a trajectory from $x^{\prime}$ outside to $x^{\prime \prime}$ inside the domain. We have shown that for $x^{\prime \prime}=-x^{\prime}$ the provision holds, however, for other schemes, e.g., $x^{\prime \prime}=-\alpha x^{\prime}(\alpha \neq 1)$, the provision (6.3) fails in general, though (6.2) still holds. On the other hand, the differential form of the flux, (1.4), has to be obtained from (6.1) in the limit $\Delta t \rightarrow 0$, which is not the case for $p_{\Delta t}(y, t)$, though it is for $p_{O U T}(y, t)$. This shows up in spades in the multi-dimensional case, because although (6.3) holds for any direction of reflection, yet the differential form of the flux is obtained in the limit only if the correct direction of oblique reflection is chosen.

The generalization of the multi-dimensional case to domains with curved boundaries and to a variable diffusion tensor $\boldsymbol{\sigma}(\boldsymbol{x}, t)$ is not straightforward and will be done separately. Note that if the diffusion tensor is constant, but un-isotropic, a local orthogonal mapping of the boundary to a plane converts the diffusion tensor from constant to variable, as can be seen from Itô's formula. However, as mentioned in Section 1 in the most common case of constant isotropic diffusion, our result extends to domains with curved boundaries, because the mapping leaves the Laplacian unchanged, though the drift changes according to Itô's formula. In this case the vector $\boldsymbol{v}$ coincides with the normal $\boldsymbol{n}$.

\section{REFERENCES}

[1] G. Lamm And K. Schulten, Extended Brownian dynamics. II. Reactive, nonlinear diffusion, J. Chem. Phys., 78 (5) (1983), pp. 2713-2734.

[2] R. ZwANZIG, Diffusion-controlled ligand binding to spheres partially covered by receptors: An effective medium treatment, Proc. Natl. Acad. Sci. U.S.A., 87 (1990), pp. 5856-5857.

[3] K. Tai, S. D. Bond, H. R. Macmillan, N. A. Baker, M. J. Holst, and J. A. McCammon, Finite Element Simulations of Acetylcholine Diffusion in Neuromuscular Junctions, Biophy. J., 84 (2003), pp. 2234-2241.

[4] L. Batsilas, A.M. Berezhrovskit, and S.Y. Shvartsman, Stochastic Model of Autocrine and Paracrine Signals in Cell Culture Assays, Biophys. J., 85 (2003), pp. 3659-3665.

[5] A. M. Berezhkovskit, Y. A. Makhnovskit, M. I. Monine, V. Yu. Zitserman, S. Y. ShvartsMAN, Boundary homogenization for trapping by patchy surfaces, J. Chem. Phys., 121 (22) (2004), pp .11390-11394.

[6] M. I. Monine And J. M. Haugh, Reactions on cell membranes: Comparison of continuum theory and Brownian dynamics simulations, J. Chem. Phys., 123 (2005), 074908.

[7] Y. Song, Y. Zhang, T. Shen, C. L. Bajaj, J. A. McCammon, and N. A. Baker, Finite Element Solution of the Steady-State Smoluchowski Equation for Rate Constant Calculations, Biophys. J., 86 (2004), pp. 2017-2029.

[8] R. ERban And J. Chapman, Reactive boundary conditions for stochastic simulations of reactiondiffusion processes, Phys. Biol., 4 (2007), pp. 16-28.

[9] S. ANDREws AND D. BRAY, Stochastic simulation of chemical reactions with spatial resolution and single molecule detail, Phys. Biol., 1 (2004) pp. 137151.

[10] W. Feller, Diffusion processes in one dimension, Trans. AMS, 77 (1) (1954), pp. 1-31.

[11] A. V. Skoroknod, Stochastic equations for diffusion processes in a bounded region, Theory of Probability and Applications, 6 (3) (1961), pp. 264-274.

[12] S. Asmussen, P. Glynn, And J. Pitman, Discretization Error in Simulation of One-Dimensional Reflecting Brownian Motion Ann. App. Prob., 5 (4) (1995), pp. 875-896.

[13] D. LÉPINGLE, Euler scheme for reflected stochastic differential equations, Mathematics and Computers in Simulation, 38 (1995), pp. 119-126.

[14] C. Costantini, B. Pacchiarotti, and F. Sartoretto, Numerical approximation for functionals of reflecting diffusion processes, SIAM J. Appl. Math., 58 (1) (1998), pp. 73-102.

[15] A. Marchewka and Z. Schuss Path integral approach to the Schrödinger current, Phys. Rev. A, 61 (2000) 052107.

[16] A. Singer And Z. Schuss, Brownian simulations and unidirectional flux in diffusion, Phys. Rev. E, $71,(2005) 026115$.

[17] C. Knessl, B. J. Matkowsky, Z. Schuss, and C. Tier, An asymptotic theory of large deviations for Markov jump processes, SIAM J. Appl. Math. 46 (6) (1985), pp. 1006-1028.

[18] C. Knessl, B. J. Matkowsky, Z. Schuss, and C. Tier, A singular perturbation approach to first passage times for Markov jump processes, J. Stat. Phys. 42 (1/2) (1986), pp.169-184.

[19] C. Knessl, B. J. Matkowsky, Z. Schuss, And C. Tier, Boundary behavior of diffusion approximations to Markov jump processes, J. Stat. Phys. 45 (1/2) (1986), pp.245-266.

[20] E. Gobet, Euler schemes and half-space approximation for the simulation of diffusion in a domain, 
ESAIM Probability and Statistics, 5 (2001), pp. 261-297.

[21] M. Bossy, E. Gobet, And D. TAlay, A symmetrized Euler scheme for an efficient approximation of reflected diffusions, J. Appl. Prob. 41, pp.877-889 (2004).

[22] F. C. Collins and G. E. Kimball, Diffusion-controlled reaction rates, J. Colloid Sci., 4 (1949), pp. 425-437.

[23] F. C. Goodrich, Random walk with semiadsorbing barrier, J. Chem. Phys., 22 (1954), pp. 588-594.

[24] A. F. Ghoniem And F. S. Sherman, Grid free simulation of diffusion using random walk methods, J. Comp. Phys., 61 (1985), pp. 1-37.

[25] S. H. Northrup, M. S. Curvin, S. A. Allison, and J. A. McCammon, Optimization of Brownian dynamics methods for diffusion-influenced rate constant calculations, J. Chem. Phys., 84 (4), (1986) pp. 2196-2203

[26] P. SzymCZAK AND A. J. C. LADD, Stochastic boundary conditions to the convection-diffusion equation including chemical reactions at solid surfaces, Phys. Rev. E, 69, 036704 (2004).

[27] N. J. B. Green, On the simulation of diffusion processes close to boundaries, Molecular Physics, 65 (6), (1988), pp. 1399-1408.

[28] P. SZYMCZAK AND A. J. C. LADD, Boundary conditions for stochastic solutions of the convectiondiffusion equation, Phys. Rev. E., 68 (2003) 036704.

[29] M. Becchria, G. Curci, And A. Vicere, Numerical solutions of first-exit-time problems, Phys. Rev E., 48 (2) (1993), pp. 1539-1546.

[30] J. Honerkamp, Stochastic Dynamical Systems: Concepts, Numerical Methods, Data Analysis, VCH Publishers, New York, 1994.

[31] R. Mannella, Absorbing boundaries and optimal stopping in a stochastic differential equation, Phys. Lett. A., 254 (5) (1999), pp. 257-262.

[32] R. Mannella, Integration of Stochastic Differential Equations on a Computer, International Journal of Modern Physics C, 13 (9) (2002), pp. 1177-1194.

[33] P. Clifford and N. J. B. Green, On the simulation of the Smoluchowski boundary condition and the interpolation of brownian paths, Molecular Physics, 57 (1), (1986), pp. 123-128.

[34] E. A. J. F. Peters and Th. M. A. O. M. Barenbrug, Efficient Brownian dynamics simulation of particles near walls. I. Reflecting and absorbing walls, Phys. Rev. E, 66 (2002), 056701.

[35] J. B. Keller and D. W. McLaughlin, The Feynman Integral, The Mathematical Monthly, 82 (5) (1975), pp. 451-576.

[36] G. H. Bryan, Note on a Problem in the Linear Conduction of Heat, Proc. Camb. Phil. Soc., 7 (1891), pp. 246-248.

[37] H. S. Carslaw and J. C. Jaeger, Conduction of Heat in Solids, 2nd edition, Oxford University Press (1959).

[38] N. Agmon, Diffusion with back reaction, J. Chem. Phys., 81 (1984), pp. 2811-2817.

[39] M. R. von Smolan Smoluchowski, Phys. Zeits., 17 (1916), p. 557. 


\begin{tabular}{c|c|c}
$\Delta t$ & $n_{\text {sur }}$ & $p_{\text {sur }}-n_{\text {sur }} / n$ \\
\hline $10^{-1}$ & 7253450 & 0.0456 \\
$10^{-2}$ & 7577156 & 0.0132 \\
$10^{-3}$ & 7670969 & 0.0039 \\
$10^{-4}$ & 7698523 & 0.0011
\end{tabular}

Survival probability: the difference between the analytic value of the survival probability $p_{\text {sur }}=$ $0.77095 \ldots$ and its numerical estimation $n_{\text {sur }} / n$ decreases by roughly $\sqrt{10}$ whenever $\Delta t$ is decreased by an order of magnitude. (Parameters: $\sigma=\kappa=x_{0}=t=1, a=0, n=10^{7}$ )

\begin{tabular}{c|c|c}
$\Delta t$ & $n_{\text {sur }}$ & $p_{\text {sur }}-n_{\text {sur }} / n$ \\
\hline $10^{-1}$ & 5986662 & 0.0814708 \\
$10^{-2}$ & 6449991 & 0.0351379 \\
$10^{-3}$ & 6707318 & 0.0094052 \\
$10^{-4}$ & 6775672 & 0.0025698 \\
\multicolumn{2}{|c}{ TABLE 6.2}
\end{tabular}

Survival probability for $\boldsymbol{a}=\mathbf{0}$. The third column lists the error between the numerical value of the survival probability $p_{\text {sur }}=0.6799545$ from the solution of the FPE and its estimate $n_{\text {sur }} / n$ from the simulation. The error decreases by about $\sqrt{10}$ whenever $\Delta t$ is decreased by an order of magnitude, indicating the convergence rate $\sqrt{\Delta t}$ of the simulation.

\begin{tabular}{c|c|c}
$\Delta t$ & $n_{\text {sur }}$ & $p_{\text {sur }}-n_{\text {sur }} / n$ \\
\hline $10^{-1}$ & 2541947 & 0.1180946 \\
$10^{-2}$ & 3399528 & 0.0323365 \\
$10^{-3}$ & 3632622 & 0.0090271 \\
$10^{-4}$ & 3693905 & 0.0028988
\end{tabular}

Survival probability for $a=(-1,0)$. The third column lists the error between the numerical value of the survival probability $p_{\text {sur }}=0.3722893$ from the solution of the FPE and its estimate $n_{\text {sur }} / n$ from the simulation. 


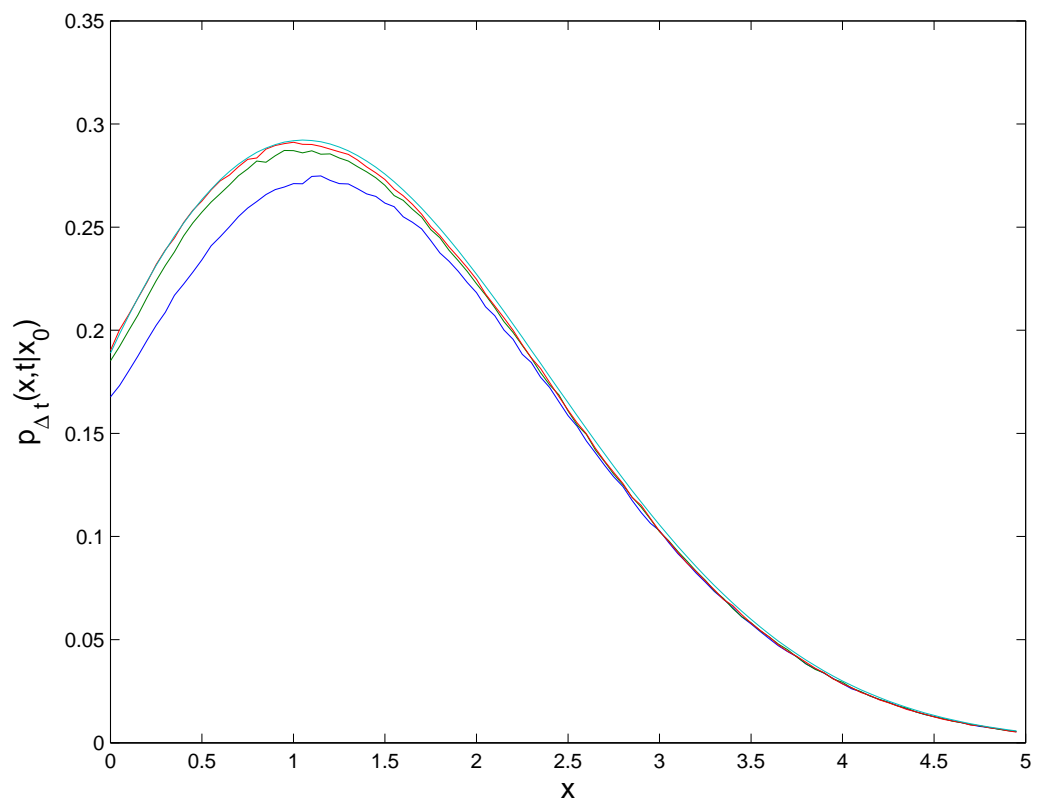

FIG. 6.1. No drift: The analytical solution (3.1) (Magenta), and the three numerical densities $\Delta t=$ $10^{-1}$ (Blue), $\Delta t=10^{-2}$ (Green), $\Delta t=10^{-3}$ (Red) approaching it from below. The numerical density of $\Delta t=10^{-4}$ is not shown, because it is difficult to distinguish it from the analytic density. (Parameters: $\sigma=\kappa=x_{0}=t=1, a=0, P=\sqrt{\pi}, n=10^{7}$ ) 


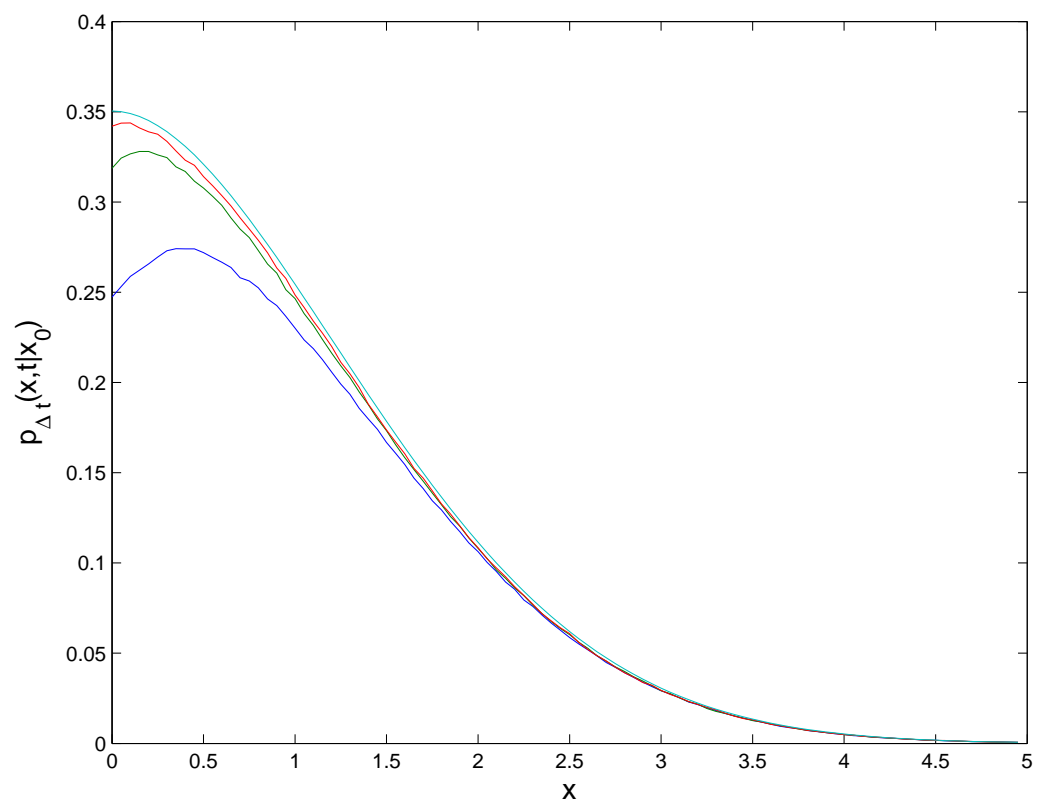

FIG. 6.2. Drift, $a=-1$ : The analytical solution 3.2) (Magenta) and the numerical densities $\Delta t=$ $10^{-1}$ (Blue), $\Delta t=10^{-2}$ (Green), $\Delta t=10^{-3}$ (Red) that approach it from below. (Parameters: $\sigma=\kappa=$ $\left.x_{0}=t=1, P=\sqrt{\pi}, n=10^{7}\right)$

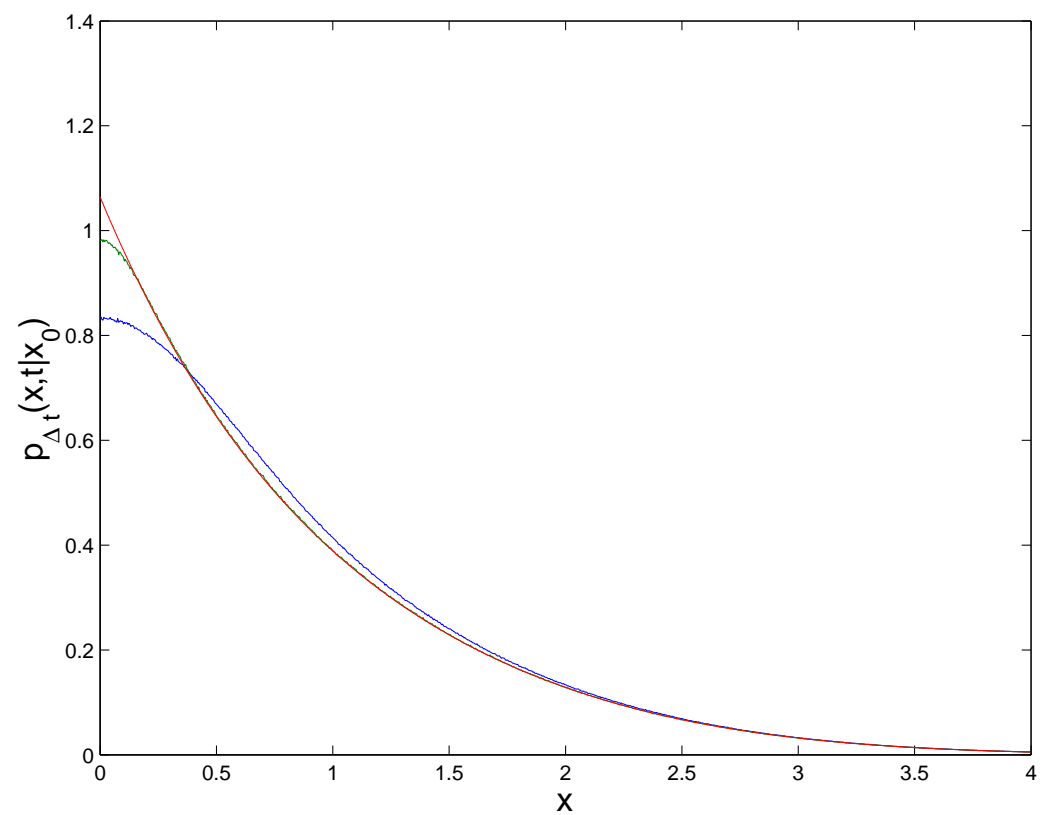

FIG. 6.3. Drift, $a=-1$, reflecting boundary $P=\kappa=0$ : The analytic solution (3.2) (Red) and the numerical densities $\Delta t=10^{-1}$ (Blue) and $\Delta t=10^{-2}$ (Green) with $n=10^{8}$ simulated trajectories to obtain a finer boundary resolution. (Parameters: $\sigma=\kappa=x_{0}=t=1$ ) 


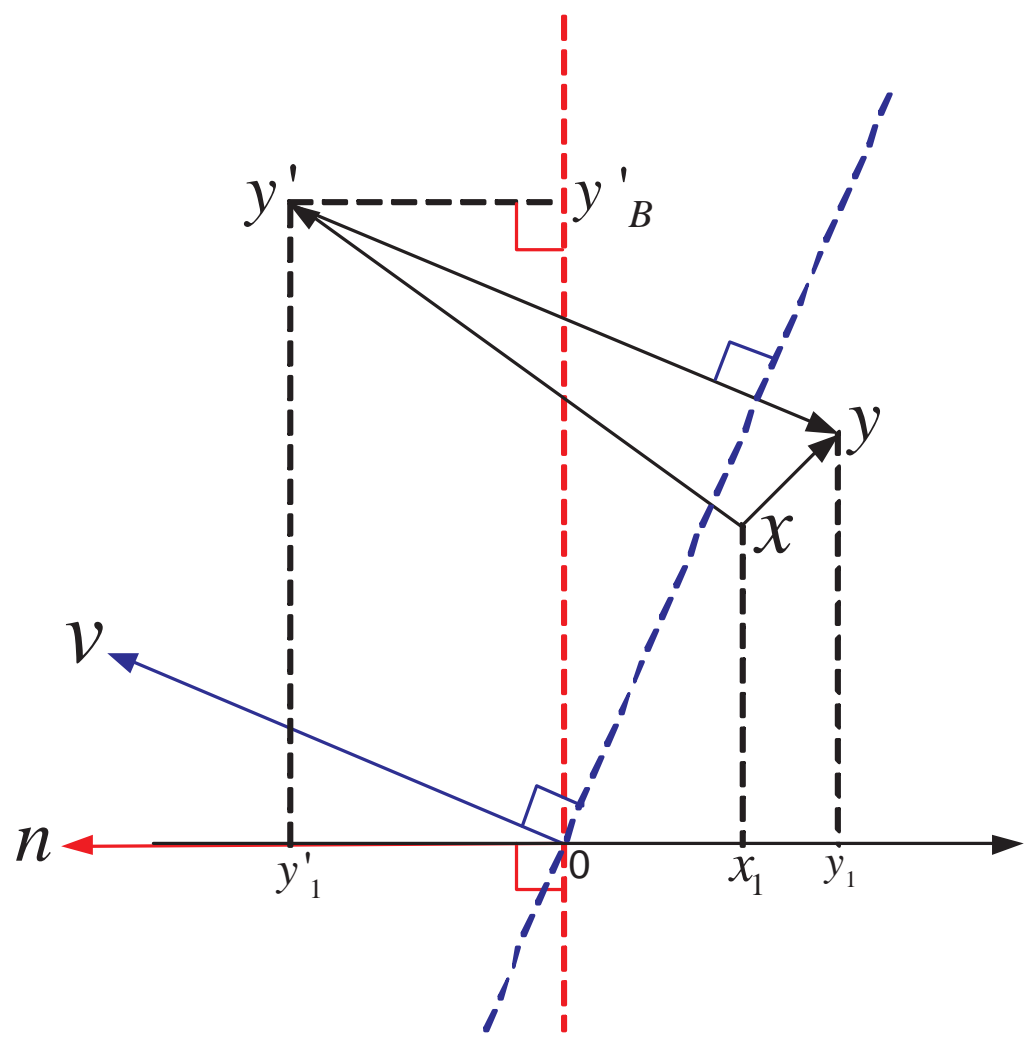

FIG. 6.4. A simulated trajectory can get from $\boldsymbol{x}$ to $\boldsymbol{y}$ in a single time step $\Delta t$ in two different ways: (i) directly from $\boldsymbol{x}$ to $\boldsymbol{y}$, without crossing the boundary, and (ii) by crossing the boundary from $\boldsymbol{x}$ to $\boldsymbol{y}^{\prime}$ and reflection in the oblique direction $\boldsymbol{v}$ with probability $1-P\left(\boldsymbol{y}_{B}^{\prime}\right) \sqrt{\Delta t}$ to $\boldsymbol{y}$. The reflection law (4.5)- 4.7) satisfies $y_{1}^{\prime}=-y_{1}$. 


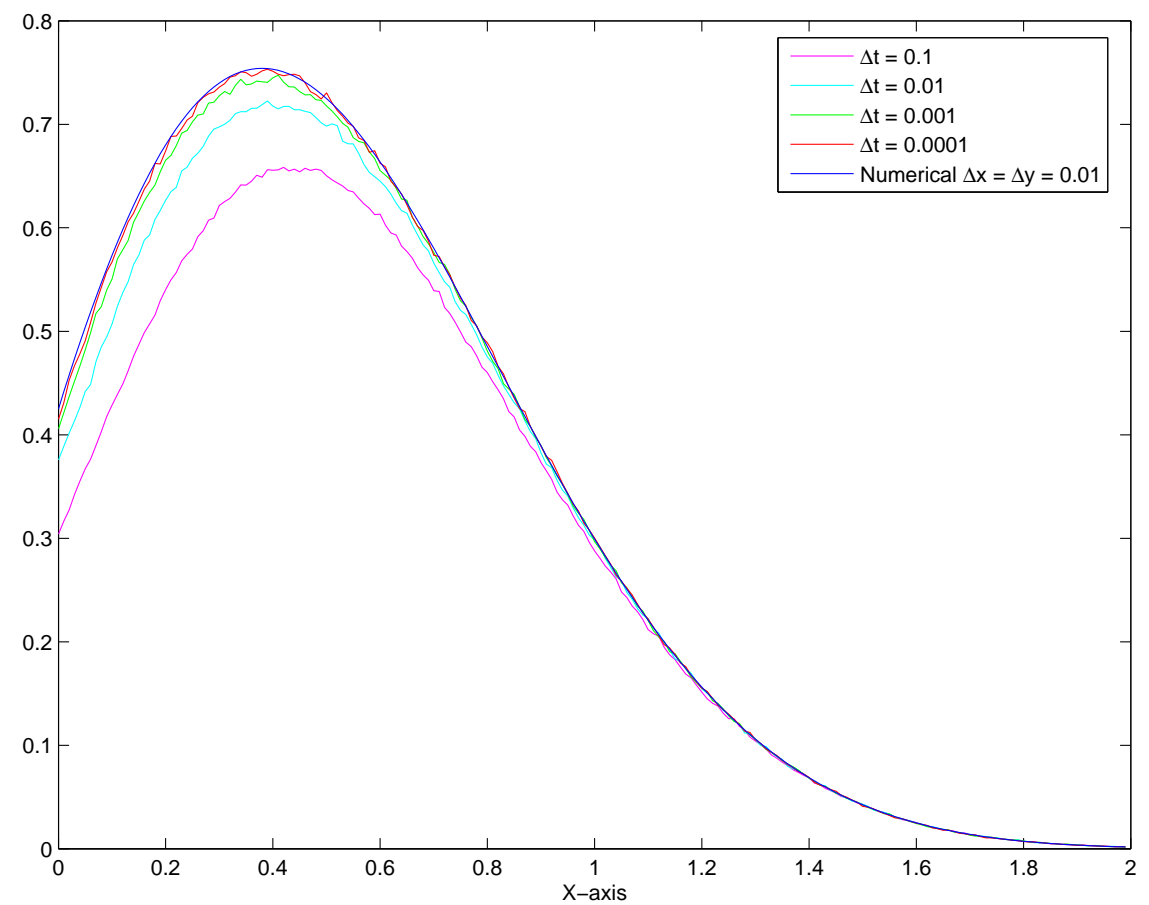

FIG. 6.5. The marginal density of $x(T)$ with no drift and correct oblique reflection (the first experiment). The numerical solution of the FPE (blue) with grid size $\Delta x=0.01$ and estimates from the simulation of $n=10^{7}$ trajectories with time steps $\Delta t=10^{-1}, 10^{-2}, 10^{-3}, 10^{-4}$. 


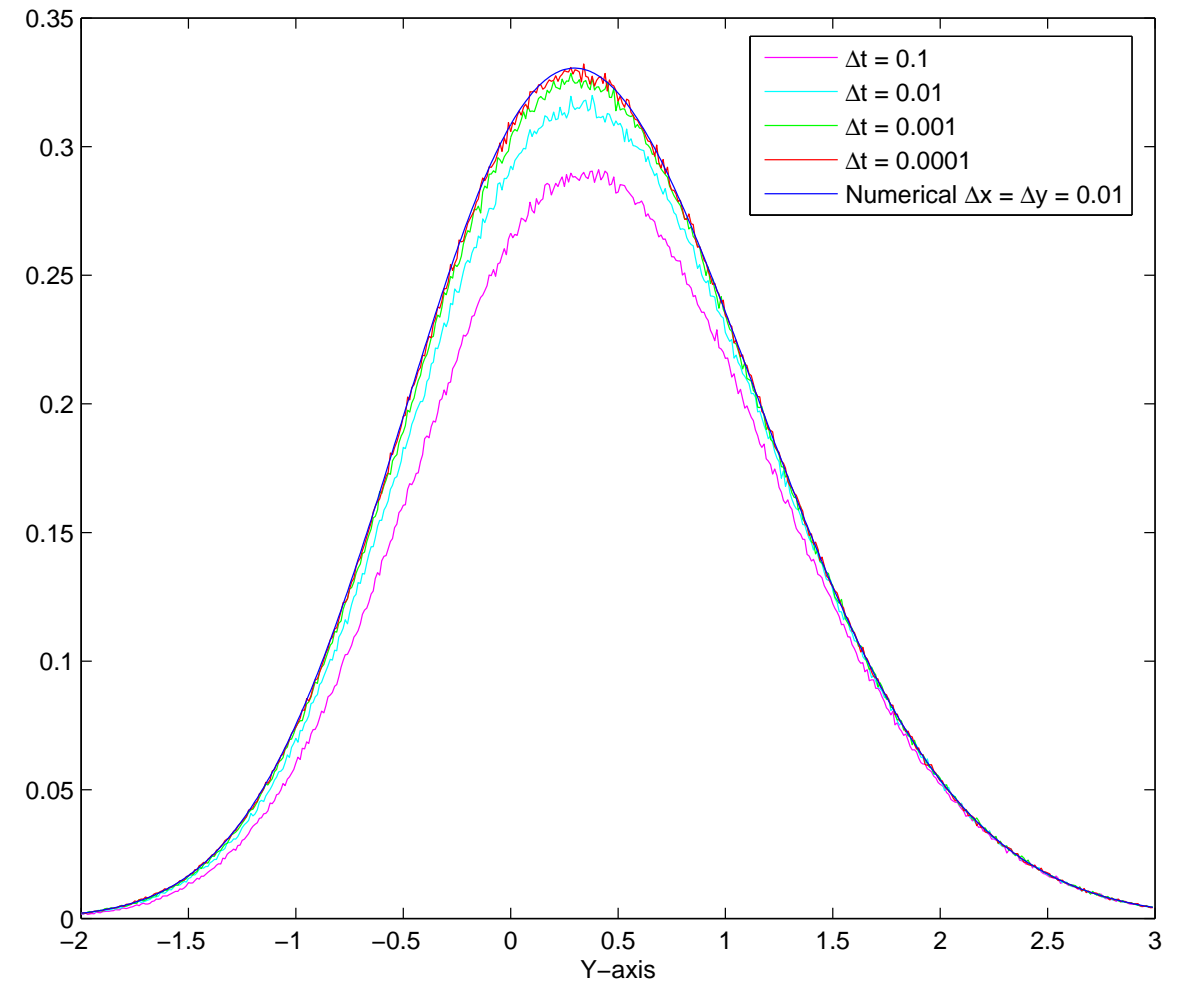

FIG. 6.6. The marginal density of $y(T)$ with no drift and correct oblique reflection (the first experiment). The numerical solution of the FPE (blue) with grid size $\Delta x=0.01$ and estimates from the simulation of $n=10^{7}$ trajectories with time steps $\Delta t=10^{-1}, 10^{-2}, 10^{-3}, 10^{-4}$. 


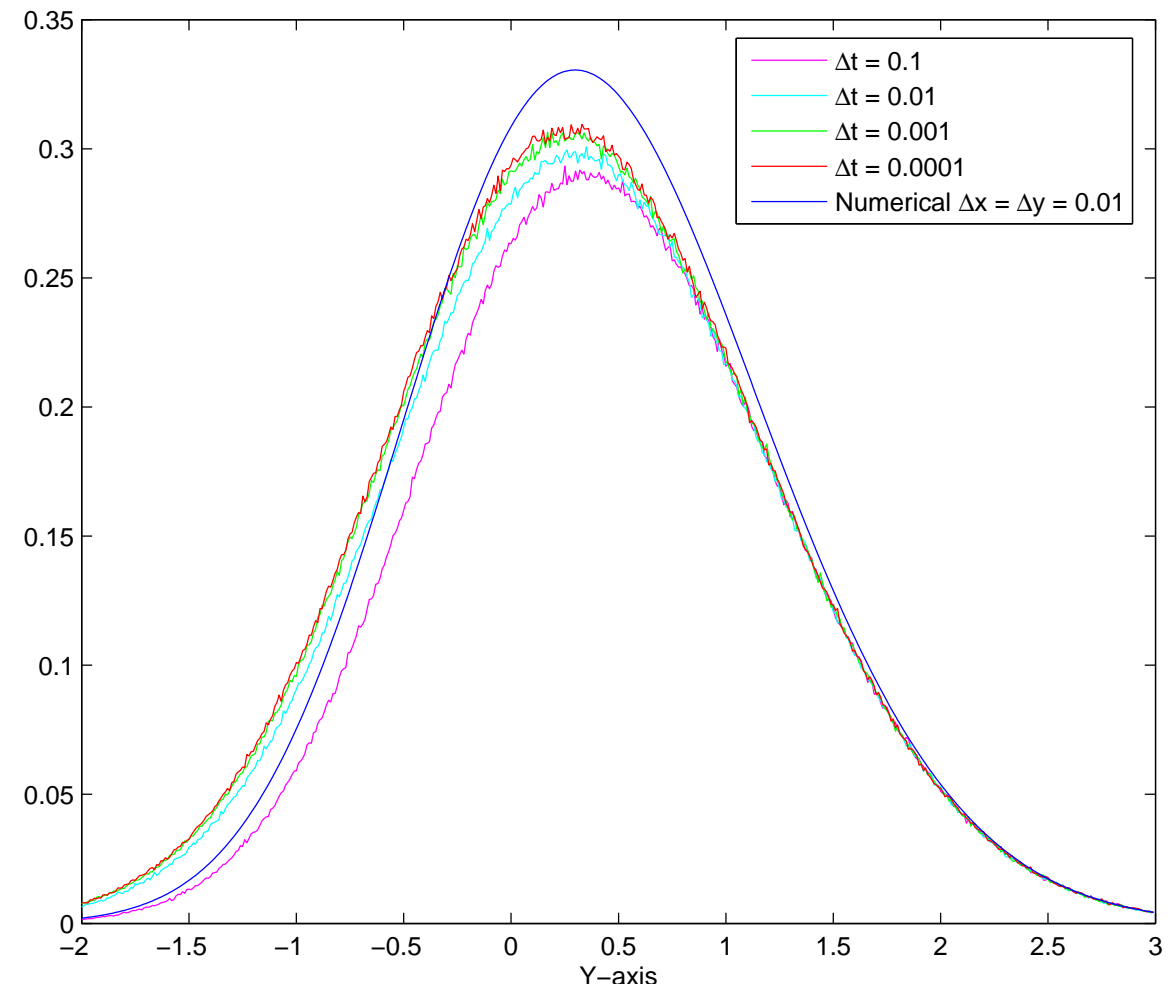

FIG. 6.7. The marginal density of $y(T)$ with no drift and with normal reflection (the second experiment). The numerical solution of the FPE (blue) with grid size $\Delta x=0.01$ and estimates from the simulation of $n=10^{7}$ trajectories with time steps $\Delta t=10^{-1}, 10^{-2}, 10^{-3}, 10^{-4}$. 


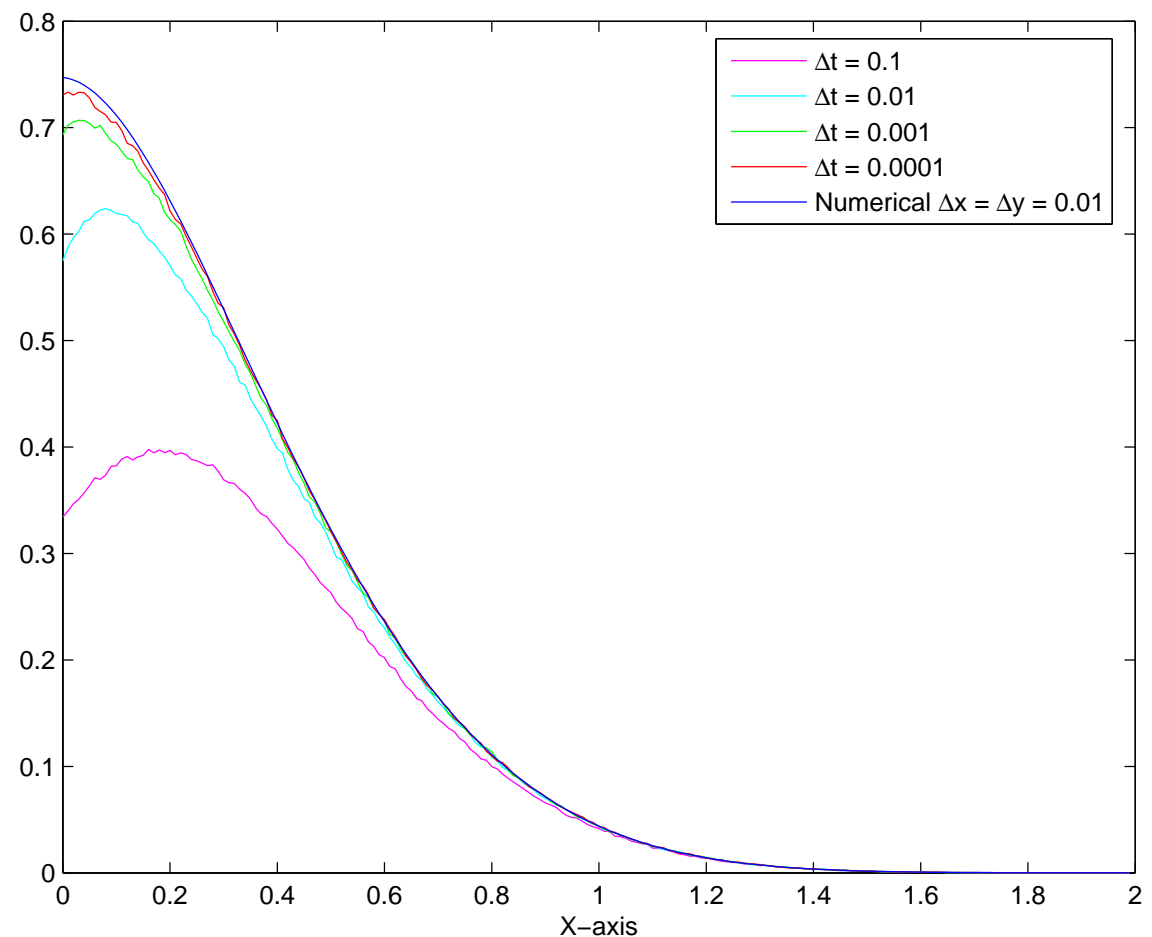

FIG. 6.8. The marginal density of $x(T)$ with drift $\boldsymbol{a}=(-1,0)$ and correct oblique reflection (the third experiment). The numerical solution of the FPE (blue) with grid size $\Delta x=0.01$ and estimates from the simulation of $n=10^{7}$ trajectories with time steps $\Delta t=10^{-1}, 10^{-2}, 10^{-3}, 10^{-4}$. 


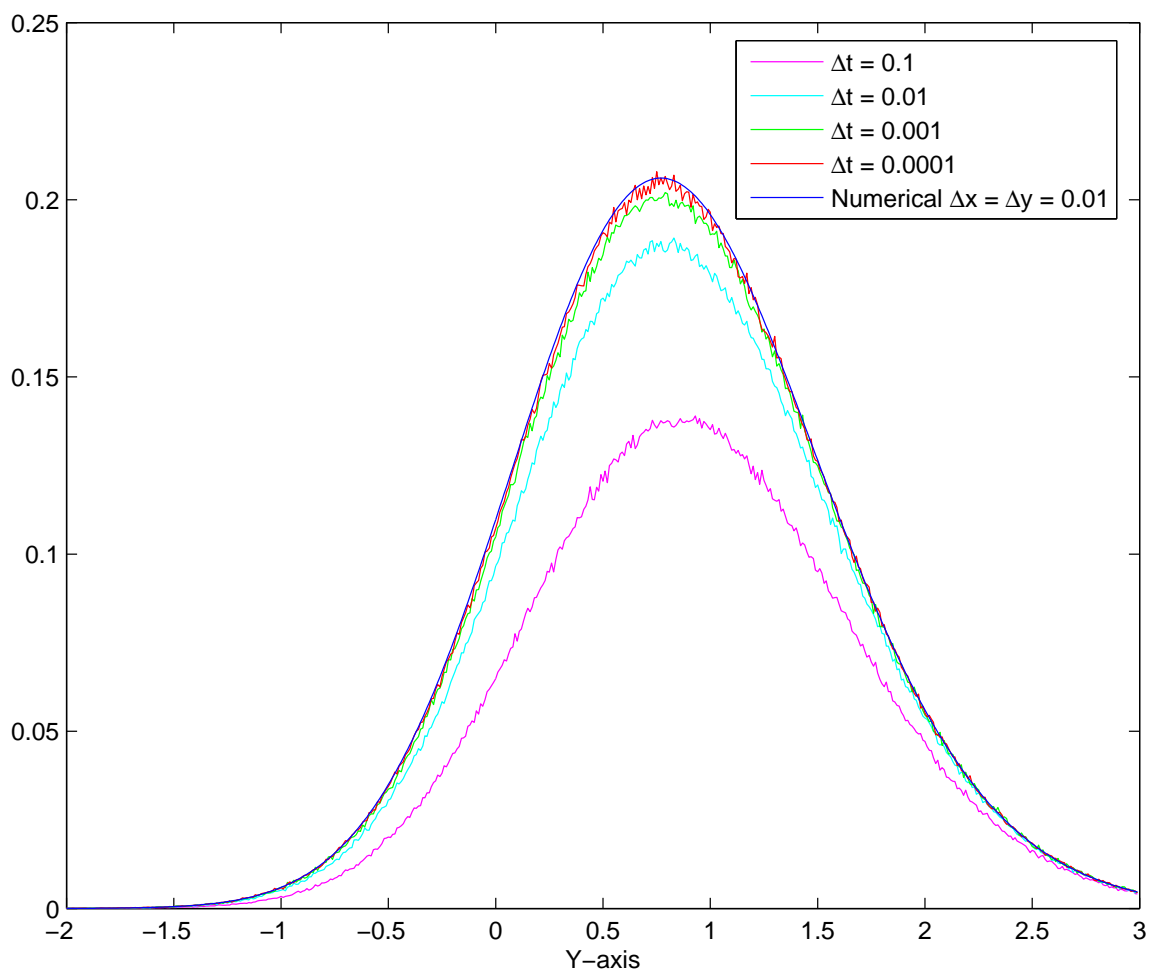

FIG. 6.9. The third experiment $\left(\boldsymbol{a}=[-1,0]^{T}\right.$, correct oblique reflection): $y$-marginal densities. The numerical solution (blue) is compared to four simulated ones (with time steps $\Delta t=10^{-1}, 10^{-2}, 10^{-3}, 10^{-4}$ ). $n=10^{7}$. Resolution: $\Delta x=0.01$. 